\title{
Gravity Waves on the Free Surface of an Incompressible Perfect Fluid of Finite Depth
}

\author{
By
}

\author{
Hideaki YosIHARA*
}

\section{§1. Introduction}

We consider the nonstationary waves on the surface of an incompressible perfect fluid of finite depth above the almost horizontal bottom in the case of two dimensional irrotational motion.

We assume that the density of mass is equal to one, the gravitational field to $(0,-1)$ and at the time $t \geqq 0$ the fluid occupies the domain

$$
\Omega(t)=\left\{\left(y_{1}, y_{2}\right) \mid y_{1} \in \boldsymbol{R}^{1},-h+b\left(y_{1}\right) \leqq y_{2} \leqq \eta\left(t, y_{1}\right)\right\}
$$

where $h$ is a positive constant. We denote by $\Gamma_{b}$ the bottom $y_{2}=-h+b\left(y_{1}\right)$ and by $\Gamma_{s}$ the free surface $y_{2}=\eta\left(t, y_{1}\right)$. The motion of the fluid occupying at $t=0$ the given domain $\Omega$ is described by the velocity $v=\left(v_{1}, v_{2}\right)$, the pressure $p$ of the fluid and $\eta$ satisfying the equations

$$
\begin{aligned}
& \frac{\partial}{\partial t} v+(v \cdot \nabla) v=-(0,1)-\nabla p \quad \text { for } t \geqq 0, y \in \Omega(t) \\
& \frac{\partial}{\partial y_{1}} v_{1}+\frac{\partial}{\partial y_{2}} v_{2}=\frac{\partial}{\partial y_{1}} v_{2}-\frac{\partial}{\partial y_{2}} v_{1}=0 \quad \text { for } t \geqq 0, y \in \Omega(t) \\
& \left(\frac{\partial}{\partial t}+v \cdot \nabla\right)\left(\eta-y_{2}\right)=0, \quad p=p_{0} \quad \text { on } \Gamma_{s} \\
& v \cdot N=0 \quad \text { on } \Gamma_{b}
\end{aligned}
$$

and taking the prescribed values

$$
\eta\left(0, y_{1}\right)=\eta_{0}\left(y_{1}\right), \quad v(0, y)=v_{0}(y)
$$

where $\nabla=\operatorname{grad}, v \cdot \nabla=v_{1}\left(\partial / \partial y_{1}\right)+v_{2}\left(\partial / \partial y_{2}\right), p_{0}$ is a constant, $N$ is the outer normal to $\Gamma_{b}$ and $v_{0}$ satisfies (1.2) for $y \in \Omega$ and (1.4).

Communicated by S. Matsuura, September 16, 1980.

* Department of Mathematics, Kyoto University, Kyoto 606, Japan. 
For the investigation of the solvability of this problem, it is convenient to use the Lagrangian coordinates. Let

$$
y_{1}=x+X_{1}(t, x), \quad y_{2}=X_{2}(t, x), \quad x \in \boldsymbol{R}^{1}
$$

be the parameter-representation of the free surface $y_{2}=\eta\left(t, y_{1}\right)$ such that

$$
\frac{\partial}{\partial t} X(t, x)=v\left(t, x+X_{1}(t, x), X_{2}(t, x)\right) \text {. }
$$

We see that on the free surface $X_{t t}=v_{t}+(v \cdot \nabla) v=-(0,1)-\nabla p$. On the other hand, differentiating $p\left(t, x+X_{1}(t, x), X_{2}(t, x)\right)=p_{0}$ with respect to $x$, we have $\left(1+X_{1 x}, X_{2 x}\right) \cdot \nabla p=0$. Hence we have $\left(1+X_{1 x}\right) X_{1 t t}+X_{2 x}\left(1+X_{2 t t}\right)=0$. It follows from (1.2) and (1.4) that under the appropriate assumptions on $v$ and $\Omega(t),\left.v_{2}\right|_{\Gamma_{s}}$ is uniquely determined by $\left.v_{1}\right|_{\Gamma_{s}}$. Therefore we conclude that there exists the operator $K=K(X, b, h)$ such that $X_{2 t}=K X_{1 t}$. In Section 3 we shall give the operator $K$ the explicit form which enables us to investigate how the operator $K$ depends on $X, b$ and $h$. In Section 4 the properties of the operator $K$ will be shown. Thus the problem is reduced to the initial value problem

(1.7) $X=U, \quad X_{1 t}=V, \quad t=0$.

In this paper we shall show that this problem is uniquely solvable in a Sobolev space when $U, V, T$ and $b$ are small. The proof is based on the quasilinearization of (1.6) and the successive approximation for the obtained quasilinear system. Our proof follows that of Nalimov [1] with the modifications caused by the fact that the operator

$$
K(0,0, h)=-i \tanh (h D), \quad D=\frac{1}{i} \frac{d}{d x}
$$

is not an isomorphism of $H^{s}$. In Section 5 we shall show that by putting

$$
Y=X_{t t}, \quad Z=X_{x}, \quad W=(X, Y, Z), \quad W^{\prime}=\left(X, Y_{1}\right),
$$

we can reduce the problem (1.6), (1.7) to the problem

$$
\begin{aligned}
& \left\{\begin{array}{l}
X_{t t}=Y, \quad Y_{1 t t}+a(W)|D| Y_{1}=f_{1}\left(W, W_{t}^{\prime}\right), \\
Y_{2 t}=f_{2}\left(W, W_{t}^{\prime}\right), \quad Z_{j t}=f_{2+j}\left(W, W_{t}^{\prime}\right), \quad j=1,2,
\end{array}\right. \\
& W=\tilde{W}, \quad W_{t}^{\prime}=\tilde{W}_{t}^{\prime}, \quad t=0 .
\end{aligned}
$$

In applying the successive approximation to the problem (1.8), (1.9), the following initial value problem is fundamental. 


$$
\begin{aligned}
& u_{t t}+a(W)|D| u=g, \quad 0 \leqq t \leqq T, \\
& u=u_{0}, \quad u_{t}=u_{1}, \quad t=0 .
\end{aligned}
$$

In Section 6 we shall deal with the initial value problems for these linear and nonlinear equations.

In the case of the infinite depth, i.e., $h=\infty$, V. I. Nalimov [1] showed the unique solvability of (1.6), (1.7) in a Sobolev space. The unique solvability of the problem on the irrotational motion of the incompressible perfect fluid with the free surface has been proved in the class of functions analytic with respect to space variables; in the case of the finite depth in two dimensions, see [2], [3], where the shallow water theory is treated; in three dimensions, see [4], [5].

We turn the reader's attention to that we do not distinguish the inessential positive constants occurring in proofs and use the same symbol $C$.

Finally I wish to thank T. Nishida who communicated the problem to me and T. Kano for the fruitful discussion with him.

\section{§2. Operators in Sobolev Spaces}

In this section we give the results of the functional analysis which will be required in later sections. In solving the problems stated in Section 1, we use only the spaces of real-valued functions of one variable, but here we deal with complex-valued functions of several variables except the last article.

2.1. Notations and Definitions. Let $k \geqq 0$ be an integer, $0<T<\infty$ and $B$ be a Banach space. We say that $u \in C^{k}([0, T], B)$ if $u$ is a $B$-valued $k$-times continuously differentiable function on $[0, T]$. Let $\Omega$ be an open set in $\mathbb{R}^{n}$. By $C^{k}(\Omega), 0 \leqq k \leqq \infty$, we denote the set of all functions defined in $\Omega$, which have continuous partial derivatives of order $\leqq k$. By $C_{0}^{k}(\Omega)$ we denote the totality of $u \in C^{k}(\Omega)$ whose support is compact in $\Omega$. By $\mathscr{B}^{k+r}(\Omega),(k \geqq 0$ is an integer, $0 \leqq r<1$, we denote the set of all $u \in C^{k}(\Omega)$ with

$$
\|u\|_{\mathscr{B}^{k+r}(\Omega)}=\sup _{|\alpha| \leqq k, x \in \Omega}\left|D^{\alpha} u(x)\right|+\sup _{|\alpha|=k, x, y \in \Omega}\left|D^{\alpha} u(x)-D^{\alpha} u(y)\right||x-y|^{-r}<\infty
$$

where $\alpha=\left(\alpha_{1}, \ldots, \alpha_{n}\right), \alpha_{j} \geqq 0$ is an integer, $|\alpha|=\alpha_{1}+\cdots+\alpha_{n}, D_{j}=(1 / i) \partial / \partial x_{j}, D^{\alpha}$ $=D_{1}^{\alpha_{1}} \ldots D_{n}^{\alpha_{n}}$. By $\mathscr{S}\left(\boldsymbol{R}^{n}\right)$ we denote the set of $u \in C^{\infty}\left(\mathbb{R}^{n}\right)$ such that

$$
\sup _{x}\left|x^{\alpha} D^{\beta} u(x)\right|<\infty
$$

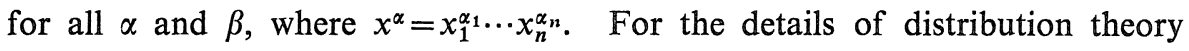


$\left(\mathscr{S}\left(\boldsymbol{R}^{n}\right), \mathscr{S}^{\prime}\left(\boldsymbol{R}^{n}\right)\right.$, Fourier transformation, convolution and others), see [6], [8]. We denote by $H^{s}\left(\boldsymbol{R}^{n}\right),-\infty<s<+\infty$, the set of all $u \in \mathscr{S}^{\prime}\left(\boldsymbol{R}^{n}\right)$ such that $(1+|\xi|)^{s} \hat{u}(\xi) \in L_{2}\left(\boldsymbol{R}^{n}\right) . \quad H^{s}\left(\boldsymbol{R}^{n}\right)$ is a Hilbert space with the inner product

$$
(u, v)_{s}=(2 \pi)^{-n} \int(1+|\xi|)^{2 s} \hat{u}(\xi) \overline{\hat{v}(\xi)} d \xi, \quad \hat{u}(\xi)=\int u(x) e^{-i x \xi} d x .
$$

We put $\|u\|_{s}=\sqrt{(u, u)_{s}},(u, v)=(u, v)_{0},\|u\|=\|u\|_{0}$. Note that

$$
(u, v)=(2 \pi)^{-n} \int \hat{u}(\xi) \overline{\hat{v}(\xi)} d \xi=\int u(x) \overline{v(x)} d x, \quad \text { (Parseval's formula). }
$$

For $u=\left(u_{1}, \ldots, u_{m}\right)$, if $u_{j} \in H^{s}\left(\boldsymbol{R}^{n}\right), j=1, \ldots, m$, then we say that $u \in H^{s}\left(\boldsymbol{R}^{n}\right)$ and write $(u, v)_{s}=\left(u_{1}, v_{1}\right)+\cdots+\left(u_{m}, v_{m}\right),\|u\|_{s}=\sqrt{(u, u)_{s}}$ for $u, v \in H^{s}\left(\boldsymbol{R}^{n}\right) . \quad \mathrm{A}$ pseudo-differential operator $P(D)$ with a symbol $P(\xi)$ is defined by

$$
P(D) u=(2 \pi)^{-n} \int P(\xi) \hat{u}(\xi) e^{i x \xi} d \xi
$$

Note that $\|u\|_{s}=\left\|(1+|D|)^{s} u\right\|$. For the convolution $u * v(x)=\int u(x-y) v(y) d y$ note that $\widehat{u * v}=\hat{u} \hat{v}, \widehat{u v}=(2 \pi)^{-n} \hat{u} * \hat{v}$.

\subsection{Convolution and Mollifier.}

Lemma 2.1 (Hausdorff-Young's inequality). Let $1 \leqq p \leqq q \leqq \infty, 1-(1 / p)$ $+(1 / q)=1 / r . \quad$ Then for $f \in L_{r}\left(\boldsymbol{R}^{n}\right), g \in L_{p}\left(\boldsymbol{R}^{n}\right)$ the inequality

$$
\|f * g\|_{L_{q}\left(R^{n}\right)} \leqq\|f\|_{L_{r}\left(R^{n}\right)}\|g\|_{L_{p}\left(R^{n}\right)}
$$

holds.

Lemma 2.2 (Hardy-Littlewood-Sobolev's inequality). Let $1<p<q<\infty$ and put $r=n(1-(1 / p)+(1 / q))$. Then for $f \in L_{p}\left(\boldsymbol{R}^{n}\right)$

$$
\left\|f *|x|^{-r}\right\|_{L_{q}\left(R^{n}\right)} \leqq K\|f\|_{L_{p}\left(R^{n}\right)}
$$

holds where $K=K(p, q, n)>0$. There is the another formulation equivalent to the above. Let $p>1, q>1$ and $(1 / p)+(1 / q)>1$ and $p u t r=n(2-(1 / p)-(1 / q))$. Then for $f \in L_{p}\left(\boldsymbol{R}^{n}\right), g \in L_{q}\left(\boldsymbol{R}^{n}\right)$

$$
\left|\int f(x) g(y)\right| x-\left.y\right|^{-r} d x d y \mid \leqq C\|f\|_{L_{p}\left(R^{n}\right)}\|g\|_{L_{q}\left(R^{n}\right)}
$$

holds where $C=C(p, q, n)>0$.

For proofs of Lemmas 2.1 and 2.2 we refer to [7] Section 2.

Take $\varphi \in C_{0}^{\infty}\left(\boldsymbol{R}^{n}\right)$ such that $\varphi=1$ in a neighbourhood of $x=0, \varphi(x) \geqq 0$ and 
$\int \varphi(x) d x=1$ and put $\varphi_{\varepsilon}(x)=\varepsilon^{-n} \varphi\left(\varepsilon^{-1} x\right), \varepsilon>0$. Since $\hat{\varphi}_{\varepsilon}(\xi)=\hat{\varphi}(\varepsilon \xi)$, we have $\left|\hat{\varphi}_{\varepsilon}(\xi)\right| \leqq 1$ and $\hat{\varphi}_{\varepsilon}(\xi) \rightarrow 1,(\varepsilon \rightarrow+0)$. Using the equality $\widehat{\varphi_{\varepsilon} * u}(\xi)=\hat{\varphi}_{\varepsilon}(\xi) \hat{u}(\xi)$ and the definition of the norm $\|\cdot\|_{s}$, we have

Lemma 2.3. Let $-\infty<s<+\infty$. Then for $u \in H^{s}\left(\mathbb{R}^{n}\right)$ we have $\left\|\varphi_{\varepsilon} * u\right\|_{s}$ $\leqq\|u\|_{s}$ and $\left\|\varphi_{\varepsilon} * u-u\right\|_{s} \rightarrow 0$ when $\varepsilon \rightarrow+0$.

Lemma 2.4 (see [6] Lemma 6.1). Let $a \in \mathscr{B}^{1}\left(\boldsymbol{R}^{n}\right)$ and define $A_{\varepsilon} b y$

$$
A_{\varepsilon} u=\varphi_{\varepsilon} *\left(a \frac{\partial u}{\partial x_{j}}\right)-a\left(\varphi_{\varepsilon} * \frac{\partial u}{\partial x_{j}}\right)
$$

Then for $u \in L_{2}\left(\boldsymbol{R}^{n}\right)$ we have

$$
\left\|A_{\varepsilon} u\right\| \leqq C\|u\|, \quad\left\|A_{\varepsilon} u-u\right\| \rightarrow 0, \quad(\varepsilon \rightarrow+0)
$$

where $C>0$ is independent of $u$ and $\varepsilon>0$.

2.3. Sobolev Spaces. Here we pick up the several facts which we shall use in estimating integral operators. For the proofs of them, see [6] Chapter 7.

\section{Lemma 2.5.}

1) $H^{s}\left(\boldsymbol{R}^{n}\right) \subseteq L_{p}\left(\boldsymbol{R}^{n}\right), \quad 0 \leqq s<\frac{n}{2}, \quad \frac{1}{p}=\frac{1}{2}-\frac{s}{n}=\frac{1}{n}\left(\frac{n}{2}-s\right)$,

i.e., there exists a constant $C>0$ such that $\|u\|_{L_{p}\left(\mathbb{R}^{n}\right)} \leqq C\|u\|_{s}$ for any $u \in H^{s}\left(\boldsymbol{R}^{n}\right)$.

2) $\quad H^{s}\left(\boldsymbol{R}^{n}\right) \hookrightarrow \mathscr{B}^{r}\left(\boldsymbol{R}^{n}\right), \frac{n}{2}<s, \quad 0 \leqq r<s-\frac{n}{2}, \quad r<1$.

\section{Corollary 2.6.}

$$
H^{s}\left(\boldsymbol{R}^{n}\right) \hookrightarrow L_{p}\left(\boldsymbol{R}^{n}\right), \quad \frac{n}{2}<s, \quad 2 \leqq p \leqq \infty
$$

Lemma 2.7 (see [8] Lemma 2.6.1). For $0<s<1$, there exists $A=A(s, n)$ $>0$ such that for any $u \in \mathscr{S}\left(\boldsymbol{R}^{n}\right)$

$$
(2 \pi)^{-n} \int|\hat{u}(\xi)|^{2}\left(1+|\xi|^{2 s}\right) d \xi=\int|u(x)|^{2} d x+A \iint|u(x+y)-u(x)|^{2}|y|^{-n-2 s} d x d y .
$$

Moreover

$$
2^{-2 s}\|u\|_{s}^{2} \leqq(2 \pi)^{-n} \int|\hat{u}(\xi)|^{2}\left(1+|\xi|^{2 s}\right) d \xi \leqq 2\|u\|_{s}^{2}
$$

Remark 2.8. Let $0<s<1,-\infty<r<+\infty$. Since $\|u\|_{r+s}=\left\|(1+|D|)^{r} u\right\|_{s}$ and 


$$
\int|y|^{-n} d y \int|u(x+y)-u(x)|^{2}|y|^{-2 s} d x=\int\left\|(u(\cdot+y)-u(\cdot))|y|^{-s}\right\|_{L_{2}\left(R^{n}\right)}^{2}|y|^{-n} d y,
$$
the norm $\|u\|_{r+s}$ is equivalent to the norm

$$
\|u\|_{r}+\left(\int_{|y| \leqq 1}\left\|(u(\cdot+y)-u(\cdot))|y|^{-s}\right\|_{r}^{2}|y|^{-n} d y\right)^{1 / 2} .
$$

Lemma 2.9. For any integer $m \geqq[n / 2]+1, u_{j} \in H^{m}\left(\boldsymbol{R}^{n}\right), j=1, \ldots, l$, and multi-indices $v_{j}, j=1, \ldots, l,\left|v_{1}\right|+\cdots+\left|v_{l}\right| \leqq m$, the estimate

$$
\left\|\left(D^{v_{1}} u_{1}\right) \cdots\left(D^{v_{l}} u_{l}\right)\right\| \leqq C\left\|u_{1}\right\|_{m} \cdots\left\|u_{l}\right\|_{m}
$$

holds where $C>0$ is a constant depending only on $n, m, l . H^{m}\left(\boldsymbol{R}^{n}\right)$ is an algebra, i.e., if $u, v \in H^{m}\left(\boldsymbol{R}^{n}\right)$ then $u v \in H^{m}\left(\boldsymbol{R}^{n}\right)$ and $\|u v\|_{m} \leqq C\|u\|_{m}\|v\|_{m}$.

Remark 2.10. By Remark 2.8 and simple calculations, we have the estimate

$$
\left\|\left(D^{v_{1}} u_{1}\right) \cdots\left(D^{v^{l}} u_{l}\right)\right\|_{r} \leqq C\left\|u_{1}\right\|_{m+r} \cdots\left\|u_{l}\right\|_{m+r}
$$

for $u_{j} \in H^{m+r}\left(\boldsymbol{R}^{n}\right), 0<r<1$. Therefore $H^{s}\left(\boldsymbol{R}^{n}\right)$ is an algebra for any real $s \geqq[n / 2]+1$. Let $u, v \in \mathscr{S}\left(\boldsymbol{R}^{n}\right), s>n / 2$. From

$$
(1+|\xi|)^{s} \widehat{u v}(\xi)=(2 \pi)^{-n} \int(1+|\xi|)^{s} \hat{u}(\xi-\eta) \hat{v}(\eta) d \eta
$$

and $(1+|\xi|)^{s} \leqq(1+|\xi-\eta|+|\eta|)^{s} \leqq 2^{s}(1+|\xi-\eta|)^{s}+2^{s}(1+|\eta|)^{s}$ we obtain by Lemma 2.1

$$
\begin{aligned}
\|u v\|_{s} \leqq C\|u\|_{s} \int & (1+|\eta|)^{s}|\hat{v}(\eta)|(1+|\eta|)^{-s} d \eta \\
& +C\|v\|_{s} \int(1+|\xi|)^{s}|\hat{u}(\xi)|(1+|\xi|)^{-s} d \xi \leqq C\|u\|_{s}\|v\|_{s} .
\end{aligned}
$$

Consequently $H^{s}\left(\boldsymbol{R}^{n}\right)$ is an algebra for $s>n / 2$.

Lemma 2.11. For $u, v_{j} \in H^{m}\left(\boldsymbol{R}^{n}\right), j=1, \ldots, l$, the estimate

$$
\|F(\cdot, v) u\|_{m} \leqq C\|F\|_{\mathscr{R}^{m}(\Omega)}\left(1+\|v\|_{m}\right)^{m}\|u\|_{m}
$$

holds where $m$ is an integer $\geqq[n / 2]+1, \Omega$ is an open set containing $\{(x, v(x))$ $\left.\in \boldsymbol{R}^{n} \times \mathbb{C}^{l} \mid x \in \boldsymbol{R}^{n}\right\}, F$ belongs to $\mathscr{B}^{m}(\Omega)$ and $C=C(m, n, l)>0$.

Remark 2.12. Using Remark 2.8 we obtain

$$
\|F(\cdot, v) u\|_{m+r} \leqq C\|F\|_{\mathscr{P}^{m+1}(\Omega)}\left(1+\|v\|_{m}\right)^{m}\left(1+\|v\|_{m+r}\right)\|u\|_{m+r}
$$

where $0<r<1$ and $\Omega$ is an open set containing $\left\{(x, z)\left|x \in \boldsymbol{R}^{n}, z \in \mathbb{C}^{l},\right| z \mid\right.$ $\leqq \sup |v|\}$. 
2.4. Estimates for Commutators. Here we deal with the case $n=1$, so we omit $\mathbb{R}^{1}$ in the notations. It is known that

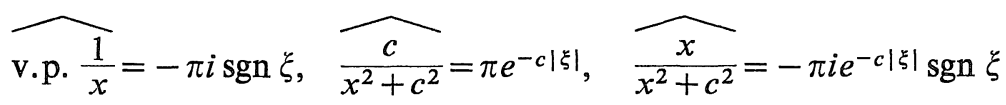
where $\operatorname{sgn} \xi=1$ for $\xi>0$, sgn $\xi=-1$ for $\xi<0$ and $c>0$.

Lemma 2.14. Let $r \geqq 0, s>1 / 2$ and $m$ be an integer $\geqq 2$. For $a, u \in \mathscr{S}$ we have

1) $\|[\operatorname{sgn} D, a] u\|_{r} \leqq C\|a\|_{r+t}\|u\|_{s-t}, \quad t \geqq 0$

2) $\left\|\left[D^{m}, a\right] u\right\| \leqq C\|a\|_{m}\|u\|_{m-1}$

3) $\left\|\left[(1+|D|)^{t}, a\right] u\right\| \leqq C\|a\|_{t}\|u\|_{t-1}, \quad t>3 / 2$

4) $\left\|\left[|D|^{t}, a\right] u\right\| \leqq C\|a\|_{1+s}\|u\|_{t-1}, \quad 0<t \leqq 1$

where $[A, B]=A B-B A$ and $C$ is $a$ constant independent of $a$ and $u$.

Proof. 1) Put $v=[\operatorname{sgn} D, a] u$. Then we have

$$
(1+|\xi|)^{r} \hat{v}(\xi)=(2 \pi)^{-1} \int(1+|\xi|)^{r}(\operatorname{sgn} \xi-\operatorname{sgn} \eta) \hat{a}(\xi-\eta) \hat{u}(\eta) d \eta .
$$

If $\operatorname{sgn} \xi-\operatorname{sgn} \eta \neq 0$ then $\operatorname{sgn} \xi=-\operatorname{sgn} \eta$ and $|\xi|+|\eta|=\xi \operatorname{sgn} \xi+\eta \operatorname{sgn} \eta=(\xi-\eta)$. sgn $\xi \leqq|\xi-\eta|$. Since $t, r \geqq 0$ we have

$$
(1+|\xi|)^{r}|\hat{v}(\xi)| \leqq \frac{1}{\pi} \int(1+|\xi-\eta|)^{r}(1+|\xi-\eta|)^{t}|\hat{a}(\xi-\eta)|(1+|\eta|)^{-t}|\hat{u}(\eta)| d \eta
$$

Taking $L_{2}$-norm with respect to $\xi$ and using Hausdorff-Young's inequality, we obtain

$$
\begin{aligned}
\|v\|_{r} & \leqq \frac{1}{\pi}\|a\|_{r+t} \int(1+|\eta|)^{-t}|\hat{u}(\eta)| d \eta \\
& \leqq \frac{1}{\pi}\|a\|_{r+t}\left(\int(1+|\eta|)^{-2 s} d \eta\right)^{1 / 2}\left(\int(1+|\eta|)^{2 s-2 t}|\hat{u}(\eta)|^{2} d \eta\right)^{1 / 2} \\
& =C\|a\|_{r+t}\|u\|_{s-t} .
\end{aligned}
$$

2) Put $v=\left[D^{m}, a\right] u$. From the estimate $\left|\xi^{m}-\eta^{m}\right| \leqq C|\xi-\eta|\left\{(1+|\xi-\eta|)^{m-1}\right.$ $\left.+(1+|\eta|)^{m-1}\right\}$ we obtain

$$
\begin{aligned}
|\hat{v}(\xi)| \leqq C \int(1+|\xi-\eta|)^{m}|\hat{a}(\xi-\eta) \hat{u}(\eta)| d \eta \\
+C \int(1+|\xi-\eta|)|\hat{a}(\xi-\eta)|(1+|\eta|)^{m-1}|\hat{u}(\eta)| d \eta
\end{aligned}
$$

Since $m \geqq 2$, we can choose $q$ such that $1 / 2<q \leqq m-1$. Therefore 


$$
\begin{aligned}
& \|v\| \leqq C\|a\|_{m} \int(1+|\eta|)^{q}|\hat{u}(\eta)|(1+|\eta|)^{-q} d \eta \\
& +C\|u\|_{m-1} \int(1+|\xi|)^{1+q}|\hat{a}(\xi)|(1+|\xi|)^{-q} d \xi \\
& \leqq C\|a\|_{m}\|u\|_{q}+C\|u\|_{m-1}\|a\|_{1+q} \leqq C\|a\|_{m}\|u\|_{m-1} \text {. }
\end{aligned}
$$

3) From $\left|(1+|\xi|)^{t}-(1+|\eta|)^{t}\right| \leqq C|\xi-\eta|\left\{(1+|\xi-\eta|)^{t-1}+(1+|\eta|)^{t-1}\right\}$ and $1 / 2<q<t-1$, we obtain 3) in the same way as in 2).

4) Put $v=\left[|D|^{t}, a\right] u$. Then we have

$$
\hat{v}(\xi)=(2 \pi)^{-1} \int\left(|\xi|^{t}-|\eta|^{t}\right)(1+|\eta|)^{1-t} \hat{a}(\zeta-\eta)(1+|\eta|)^{t-1} \hat{u}(\eta) d \eta .
$$

Noting that $0<t \leqq 1$, we estimate $f=\left.|| \xi\right|^{t}-|\eta|^{t} \mid(1+|\eta|)^{1-t}$. For $|\eta| \leqq 1$, $f \leqq C(1+|\xi-\eta|)$. For $|\eta| \geqq 1$ and $|\xi| \geqq|\eta|$,

$$
\begin{aligned}
f \leqq|\xi|^{t}\left(1-\left(\frac{|\eta|}{|\xi|}\right)^{t}\right)(2|\eta|)^{1-t} & \leqq|\xi|^{t}\left(1-\frac{|\eta|}{|\xi|}\right)(2|\eta|)^{1-t} \\
& \leqq(|\xi|-|\eta|)\left(\frac{2|\eta|}{|\xi|}\right)^{1-t} \leqq 2|\zeta-\eta| .
\end{aligned}
$$

For $|\eta| \geqq 1$ and $|\xi| \leqq|\eta|$,

$$
f \leqq\left(1-\left(\frac{|\xi|}{|\eta|}\right)^{t}\right)|\eta|^{t}(2|\eta|)^{1-t} \leqq\left(1-\frac{|\xi|}{|\eta|}\right) 2^{1-t}|\eta| \leqq 2|\xi-\eta| .
$$

Hence we have $f \leqq C(1+|\xi-\eta|)$ and

$$
|\hat{v}(\xi)| \leqq C \int(1+|\xi-\eta|)|\hat{a}(\xi-\eta)|(1+|\eta|)^{t-1}|\hat{u}(\eta)| d \eta .
$$

In the same way as in 1 ), we obtain $\|v\| \leqq C\|a\|_{1+s}\|u\|_{t-1}$. The proof is complete.

Lemma 2.15. Let $h>0, s \geqq 0$. For $u \in H^{0}$, the estimates

$$
\|(\operatorname{sgn} D-\tanh (h D)) u\|_{s} \leqq C\|u\|, \quad\left\|\left(1-\tanh ^{2}(h D)\right) u\right\|_{s} \leqq C\|u\|
$$

hold where $C=C(h, s)>0$.

Proof. Since $\tanh (h \xi)=\left(e^{h \xi}-e^{-h \xi}\right)\left(e^{h \xi}+e^{-h \xi}\right)^{-1}=(\operatorname{sgn} \xi)\left\{1-2 e^{-h|\xi|}\right.$. $\left.\left(e^{h|\xi|}+e^{-h|\xi|}\right)^{-1}\right\}$, we have

$$
|\operatorname{sgn} \xi-\tanh (h \xi)|+\left|1-\tanh ^{2}(h \xi)\right| \leqq C e^{-h|\xi|} .
$$

From this we obtain the required estimates.

Lemma 2.16. For $0<s<1$ and an integer $m \geqq 0$, there exists $A=A(s, m)$ $>0$ such that for any $u \in \mathscr{S}$ 


$$
\begin{aligned}
& (2 \pi)^{-1} \int|\hat{u}(\xi)|^{2}\left(1+|\xi|^{2 m+2 s}\right) d \xi \\
& \quad=\int|u(x)|^{2} d x+\left.A \iint\left|D_{y}^{m} u(. x+y)-u(. x)\right|^{2}|.|\right|^{1-2 s} d x d y .
\end{aligned}
$$

Moreover

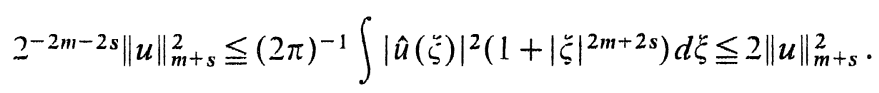

Proof. Using the Parseval's formula,

$$
\begin{aligned}
& \iint\left|D_{y}^{m} \frac{u(x+y)-u(x)}{y}\right|^{2}|y|^{1-2 s} d x d y \\
& =(2 \pi)^{-1} \int|\hat{u}(\xi)|^{2} d \xi \int\left|D_{y}^{m} \frac{\mathcal{c}^{i y^{*}}-1}{y}\right|^{2}\left|y^{\prime}\right|^{1-2 s} d y,
\end{aligned}
$$

by the transformation $y \rightarrow \xi^{-1} z$,

$$
=(2 \pi)^{-1}\left(\int|\hat{u}(\breve{\zeta})|^{2}|\xi|^{2 m+2 s} d \xi\right)\left(\int\left|D_{z}^{m} \frac{e^{i z}-1}{z}\right|^{2}|z|^{1-2 s} d z\right) .
$$

Since $\left|D_{z}^{m}\left(e^{i z}-1\right) / z\right|^{2}|z|^{1-2 s} \leqq C(1+|z|)^{-2}|z|^{1-2 s}$ and $0<s<1$, the integral

$$
\int\left|D_{z}^{m}-\frac{e^{i z}-1}{z}\right|^{2}|z|^{1-2 s} d z
$$

converges. It is obvious that if we put this integral equal to $A^{-1}$ then we obtain the equality in question. For $a>0, b>0$, we have $1+b^{a} \leqq 1+(1+b)^{a}$ $\leqq 2(1+b)^{a}$. By a substitution $a \rightarrow a^{-1}$, we have $1+b^{1 / a} \leqq 2(1+b)^{1 / a}$, and by $b \rightarrow$ $b^{a}, 1+b \leqq 2\left(1+b^{a}\right)^{1 / a}$. Hence $2^{-a}(1+b)^{a} \leqq 1+b^{a} \leqq 2(1+b)^{a}$ is valid. Putting $a=2 m+2 s, b=|\xi|$, we obtain $2^{-2 m-2 s}(1+|\xi|)^{2 m+2 s} \leqq 1+|\xi|^{2 m+2 s} \leqq 2(1+|\xi|)^{2 m+2 s}$. If we multiply these by $(2 \pi)^{-1}|\hat{u}(\xi)|^{2}$ and integrate, then we obtain the required inequality.

\section{§3. Representation of the Operator $\mathbb{K}$}

In this section we give the operator $K$ the representation which is adequate when we investigate the dependence of $K$ on functions defining the bottom and the free surface. We can not assert the validity of the following calculation if we do not indicate which space the functions under consideration belong to, but we proceed with calculations under the ambiguous assumption that all occurring functions are smooth, small and tend to zero when variables tend to the infinity. 
Let $\Omega$ be the domain in the $y_{1}, y_{2}$-space which is identified with the $z$ $=y_{1}+i y_{2}$ plane. Assume that the boundary of $\Omega$ consists of $\Gamma_{s}$ and $\Gamma_{b}$ which are given by

$$
\begin{cases}\Gamma_{s}: & \left(x+X_{1}(x), X_{2}(x)\right) \text { or } z(x)=x+X_{1}(x)+i X_{2}(x), \\ \Gamma_{b}: & (x,-h+b(x)) \text { or } w(x)=x+i(-h+b(x)), \quad-\infty<x<+\infty .\end{cases}
$$

Let $v_{1}, v_{2}$ be defined in $\Omega$ and satisfy the equations

$$
\begin{aligned}
& \frac{\partial}{\partial y_{1}} v_{1}+\frac{\partial}{\partial y_{2}} v_{2}=\frac{\partial}{\partial y_{2}} v_{1}-\frac{\partial}{\partial y_{1}} v_{2}=0 \text { in } \Omega \\
& v \cdot N=0 \text { on } \Gamma_{b} .
\end{aligned}
$$

Then $F=v_{1}-i v_{2}$ is holomorphic in $\Omega$. Put

$$
\left\{\begin{array}{l}
f(x)=f_{1}(x)+i f_{2}(x)=F(z(x)) \\
g(x)=g_{1}(x)+i g_{2}(x)=F(w(x)) \\
b_{1}(x)=\frac{d b}{d x}(x)
\end{array}\right.
$$

From $v \cdot N=0$ we have $g_{2}(x)=-b_{1}(x) g_{1}(x)$. Taking $z_{0} \in \Gamma_{s}$ and the closed path $\gamma$ in $\Omega$ and letting $\gamma \rightarrow \Gamma_{s} \cup \Gamma_{b}$, we obtain

$$
0=\frac{1}{2 \pi i} \int_{\gamma} \frac{F(z)}{z-z_{0}} d z=\frac{-\pi i}{2 \pi i} F\left(z_{0}\right)-\frac{1}{2 \pi i} \text { v.p. } \int_{\Gamma_{s}} \frac{F(z)}{z-z_{0}} d z+\frac{1}{2 \pi i} \int_{\Gamma_{b}} \frac{F(z)}{z-z_{0}} d z .
$$

Using (3.1) and (3.2) we have

$$
f(x)+\frac{1}{\pi i} \text { v.p. } \int \frac{f(y)}{z(y)-z(x)} \frac{d z(y)}{d y} d y=\frac{1}{\pi i} \int \frac{g(y)}{w(y)-z(x)} \frac{d w(y)}{d y} d y .
$$

By

$$
\begin{aligned}
\frac{1}{z(y)-z(x)} \frac{d z(y)}{d y} & =\frac{\partial}{\partial y} \log (z(y)-z(x))=\frac{\partial}{\partial y}\left(\log (y-x)+\log \frac{z(y)-z(x)}{y-x}\right) \\
& =\frac{1}{y-x}+\frac{\partial}{\partial y} \log \left(1+\frac{X_{1}(y)-X_{1}(x)}{y-x}+i \frac{X_{2}(y)-X_{2}(x)}{y-x}\right) \\
\frac{1}{w(y)-z(x)} \frac{d w(y)}{d y} & =\frac{\partial}{\partial y} \log (w(y)-z(x)) \\
& =\frac{\partial}{\partial y}\left(\log (y-x-i h)+\log \frac{w(y)-z(x)}{y-x-i h}\right) \\
& =\frac{y-x+i h}{(y-x)^{2}+h^{2}}+\frac{\partial}{\partial y} \log \left(1+\frac{-X_{1}(x)+i b(y)-i X_{2}(x)}{y-x-i h}\right)
\end{aligned}
$$

and (2.13), the real part of (3.3) becomes after the partial integration in the integrals containing $\log$, 


$$
f_{1}+i \operatorname{sgn} D f_{2}+A_{1} f_{1}+A_{2} f_{2}=e^{-h|D|} g_{1}+i \operatorname{sgn} D e^{-h|D|} g_{2}+A_{3} g_{1}+A_{4} g_{2}
$$

where

$$
\left\{\begin{aligned}
A_{j} u= & \int a_{j}(x, y) \frac{d u}{d y}(y) d y, \quad j=1,2,3,4, \\
& a_{1}=-\frac{1}{\pi} \operatorname{Im} \log \left(1+\frac{X_{1}(x)-X_{1}(y)}{x-y}+i \frac{X_{2}(x)-X_{2}(y)}{x-y}\right) \\
& a_{2}=-\frac{1}{\pi} \operatorname{Re} \log \left(1+\frac{X_{1}(x)-X_{1}(y)}{x-y}+i \frac{X_{2}(x)-X_{2}(y)}{x-y}\right) \\
& a_{3}=-\frac{1}{\pi} \operatorname{Im} \log \left(1+\frac{X_{1}(x)+i X_{2}(x)-i b(y)}{x-y+i h}\right) \\
& a_{4}=-\frac{1}{\pi} \operatorname{Re} \log \left(1+\frac{X_{1}(x)+i X_{2}(x)-i b(y)}{x-y+i h}\right) .
\end{aligned}\right.
$$

Taking $w_{0} \in \Gamma_{b}$ and proceeding in the same way as the above, we obtain

$$
g_{1}-i \operatorname{sgn} D g_{2}+A_{5} g_{1}+A_{6} g_{2}=e^{-h|D|} f_{1}-i \operatorname{sgn} D e^{-h|D|} f_{2}+A_{7} f_{1}+A_{8} f_{2}
$$

where

$$
\left\{\begin{aligned}
A_{j} u= & \int a_{j}(x, y) \frac{d u}{d y}(y) d y, \quad j=5,6,7,8, \\
& a_{5}=\frac{1}{\pi} \operatorname{Im} \log \left(1+i \frac{b(x)-b(y)}{x-y}\right) \\
& a_{6}=\frac{1}{\pi} \operatorname{Re} \log \left(1+i \frac{b(x)-b(y)}{x-y}\right) \\
& a_{7}=\frac{1}{\pi} \operatorname{Im} \log \left(1+\frac{-X_{1}(y)+i b(x)-i X_{2}(y)}{x-y-i h}\right) \\
a_{8} & =\frac{1}{\pi} \operatorname{Re} \log \left(1+\frac{-X_{1}(y)+i b(x)-i X_{2}(y)}{x-y-i h}\right) .
\end{aligned}\right.
$$

Eliminating $g_{1}, g_{2}$ from (3.4), (3.6) and $g_{2}=-b_{1} g_{1}$, we obtain

$$
\left\{1-e^{-2 h|D|}-i \operatorname{sgn} D\left(1+e^{-2 h|D|}\right) B_{2}\right\} f_{1}=-i \operatorname{sgn} D\left(1+e^{-2 h|D|}\right)\left(1+B_{1}\right) f_{2} .
$$

Since $f_{1}=v_{1}, f_{2}=-v_{2}, K=K(X)=K(X, b, h)$ can be written in the form

$$
\begin{aligned}
K & =-\left(1+B_{1}\right)^{-1}\left(i \tanh (h D)+B_{2}\right) \\
& =-i \tanh (h D)-B_{2}+B_{1}\left(1+B_{1}\right)^{-1}\left(i \tanh (h D)+B_{2}\right) \\
& =-i \tanh (h D)+K_{1}
\end{aligned}
$$

where

$$
\left\{\begin{aligned}
B_{1}= & i \operatorname{sgn} D\left(1+e^{-2 h|D|}\right)^{-1}\left\{-A_{2}+e^{-h|D|} A_{8}+B_{3}\left(-i \operatorname{sgn} D e^{-h|D|}\right.\right. \\
& \left.\left.+A_{8}\right)-\left(e^{-h|D|}+B_{3}\right) B_{4}\left(1+B_{4}\right)^{-1}\left(-i \operatorname{sgn} D e^{-h|D|}+A_{8}\right)\right\} \\
B_{2}= & i \operatorname{sgn} D\left(1+e^{-2 h|D|}\right)^{-1}\left\{A_{1}-e^{-h|D|} A_{7}-B_{3}\left(e^{-h|D|}+A_{7}\right)\right. \\
& \left.+\left(e^{-h|D|}+B_{3}\right) B_{4}\left(1+B_{4}\right)^{-1}\left(e^{-h|D|}+A_{7}\right)\right\}
\end{aligned}\right.
$$




$$
\mid \begin{aligned}
& B_{3}=-i \operatorname{sgn} D e^{-h|D|} b_{1}+A_{3}-A_{4} b_{1}, \\
& B_{4}=i \operatorname{sgn} D b_{1}+A_{5}-A_{6} b_{1}, \\
& A_{j}, j=1, \ldots, 8, \text { are defined by (3.5), (3.7) } \\
& b_{1}=\frac{d b}{d x}
\end{aligned}
$$

\section{§4. Estimates for Integral Operators}

We shall show that, roughly speaking, $K_{1}$ and $\left[\partial_{t}^{k} \partial_{x}^{j}, K\right]$ are operators of order -1 . To this end, first of all, we consider the integral operator of the form

$$
A u(x)=\int\left(\prod_{1}^{M} \frac{a_{j}(x)-a_{j}(y)}{x-y}\right) F\left(\frac{b(x)-b(y)}{x-y}\right) u(y) d y
$$

where $b=\left(b_{1}, \ldots, b_{N}\right), a_{j}, b_{j}$ are real-valued and $F$ is smooth in a neighbourhood of $0 \in \boldsymbol{R}^{N}$, or

$$
A u(x)=\int\left(\prod_{1}^{M} \frac{a_{j}(x)-b_{j}(y)}{x-y \pm i h}\right) F\left(\frac{f(x)-g(y)}{x-y \pm i h}\right) u(y) d y
$$

where $f=\left(f_{1}, \ldots, f_{N}\right), g=\left(g_{1}, \ldots, g_{N}\right)$ and $F$ is smooth in a neighbourhood of $0 \in C^{N}$.

In the following two articles we shall show that if functions $a, b, \ldots$, occurring in the kernel are in $H^{s}$, then $A u$ is in $H^{s}$ and Lipschitz continuous with respect to $a, b, \ldots$. Since $\mathscr{S}$ is dense in $H^{s}$, to simplify the statements we shall assume that, unless the contrary is stated, all functions $a, b, \ldots$, are in $\mathscr{S}$.

\subsection{Operators of the Form (4.1).}

Lemma 4.3. Let $k$ be an integer $\geqq 0, r \geqq 0$ and $s>1 / 2$. For $A$ defined by

$$
\begin{aligned}
A u(x) & =\int \frac{a(x)-a(y)}{x-y} D^{k} u(y) d y=\int \frac{a(x)-a(x-y)}{y} D^{k} u(x-y) d y \\
& =\int\left(D_{y}^{k} \frac{a(x)-a(x-y)}{y}\right) u(x-y) d y
\end{aligned}
$$

we have $\|A u\|_{r} \leqq C\|a\|_{k+r}\|u\|_{s}$ where $C=C(k, r, s)>0$.

Proof. Since

$$
\begin{aligned}
A u & =a\left(\text { v.p. } \frac{1}{x} * D^{k} u\right)-\text { v.p. } \frac{1}{x} *\left(a D^{k} u\right) \\
& =-\pi i[a, \operatorname{sgn} D] D^{k} u
\end{aligned}
$$


the lemma follows from Lemma 2.14.

Lemma 4.4. Let $j$ be an integer $\geqq 0$ and

$$
I(x)=\int\left|D_{y}^{j} \frac{a(x)-a(x-y)}{y}\right||u(x-y)| d y .
$$

Then $\|I\| \leqq C\|a\|_{j+(1 / 2)}\|u\|$ where $C=C(j)>0$.

Proof. Since

$$
\begin{aligned}
\int I(x)^{2} d x & \leqq \int d x \int\left|D_{y}^{j} a(x)-a(x-y)\right|^{2} d y \int|u(x-y)|^{2} d y \\
& =\int\left|D_{y}^{j} \frac{a(-x-y)-a(-x)}{y}\right|^{2} d x d y\|u\|^{2},
\end{aligned}
$$

we obtain the required estimate if we put $m=j, s=1 / 2$ in Lemma 2.16.

Lemma 4.5. Let $j, k$ be integers $\geqq 0, s>1 / 2$ and

$$
\left.I(x)=\int\left|D_{y}^{j} \underline{a(x)-a(x-y)}\right| \mid D_{y}^{k} b(x)-\frac{b(x-y)}{y}\right) \mid d y .
$$

Then $\|I\| \leqq C\|a\|_{j+s}\|b\|_{k+1}$ where $C=C(j, k, s)>0$.

Proof. Let $0<r<1$. Then

$$
\begin{aligned}
\left|a(x)-\frac{a(x-y)}{y}\right| & =|a(x)-a(x-y)||y|^{-r}|y|^{r-1} \\
& \leqq C\|a\|_{\mathscr{O}^{r}}(1+|y|)^{-r}|y|^{r-1} .
\end{aligned}
$$

If $m \geqq 1$ then

$$
\begin{aligned}
\partial_{y}^{m} & \frac{a(x)-a(x-y)}{y}=\partial_{y}^{m} \int_{-1}^{0} a^{(1)}(x+t y) d t=\int_{-1}^{0} t^{m} a^{(m+1)}(x+t y) d t \\
& =\int_{-1}^{0} t^{m} \frac{1}{y} \frac{\partial}{\partial t} a^{(m)}(x+t y) d t \\
& =\frac{1}{y}\left\{-(-1)^{m} a^{(m)}(x-y)-\int_{-1}^{0} m t^{m-1} a^{(m)}(x+t y) d t\right\} \\
& =\frac{m}{y} \int_{-1}^{0} t^{m-1}\left\{a^{(m)}(x-y)-a^{(m)}(x+t y)\right\} d t \\
& =-\frac{1}{y}\left\{(-1)^{m} a^{(m)}(x-y)+m \partial_{y}^{m-1} \frac{a(x)-a(x-y)}{y}\right\}
\end{aligned}
$$

where $a^{(j)}(x)=\partial_{x}^{j} a(x)$. Therefore for $s=0, r$ we have

$$
\begin{aligned}
\left|\partial_{y}^{m} \frac{a(x)-a(x-y)}{y}\right| \leqq m \mid & \left.y\right|^{-1} \int_{-1}^{0}|t|^{m-1} \mid a^{(m)}(x-y) \\
& -a^{(m)}(x+t y)|| y+\left.t y\right|^{-s}|1+t|^{s}|y|^{s} d t
\end{aligned}
$$




$$
\leqq 2 m|y|^{s-1}\left\{\int_{-1}^{0}|t|^{m-1}|t+1|^{s} d t\right\}\left\|D^{m} a\right\|_{\mathscr{B} s} .
$$

Since $0<r<1, \int_{-1}^{0}|t|^{m-1}|t+1|^{r} d t<\infty$. These inequalities and (4.6) show that

$$
\left|D_{y}^{m} \frac{a(x)-a(x-y)}{y}\right| \leqq C\left\|D^{m} a\right\|_{\mathscr{G}^{r}}(1+|y|)^{-r}|y|^{r-1}
$$

holds where $m \geqq 0$ and $C=C(m, r)>0$. From this it follows that

$$
\begin{aligned}
& \int I(x)^{2} d x=\int d x\left\{\int\left|D_{y}^{j} \frac{a(x)-a(x-y)}{y}\right|\left|D_{y}^{k} \frac{b(x)-b(x-y)}{y}\right| d y\right\}^{2} \\
& \leqq C\left\|D^{j} a\right\|_{\mathscr{B}}^{2}\left\{\int d x \int(1+|y|)^{-r}|y|^{(1 / 2)(r-1)}\right. \\
&\left.\times\left|D_{y}^{k} \frac{b(x)-b(x-y)}{y}\right||y|^{(1 / 2)-(1-(r / 2))} d y\right\}^{2} \\
& \leqq C\left\|D^{j} a\right\|_{\mathscr{B}}^{2} \int(1+|y|)^{-2 r}|y|^{r-1} d y \\
& \times \int\left|D_{y}^{k} \frac{b(x)-b(x-y)}{y}\right|^{2}|y|^{1-2(1-(r / 2))} d x d y .
\end{aligned}
$$

Let $r$ be so small that $0<r<s-(1 / 2)$. Then from Lemma 2.5 we obtain

$$
\left\|D^{j} a\right\|_{\mathscr{B} r} \leqq C\left\|D^{j} a\right\|_{s} \leqq C\|a\|_{j+s} .
$$

If we put $m=k, s=1-(r / 2)$ in Lemma 2.16 , then the integral containing $b$ is smaller than $C\|b\|_{k+(1-(r / 2))}^{2}$, which proves the lemma.

Lemma 4.8. Let $k$ be an integer $\geqq 0, s>1 / 2, b=\left(b_{1}, \ldots, b_{N}\right), b_{j}$ be realvalued and

$$
A u(x)=\int\left(D_{y}^{k} \frac{a(x)-a(x-y)}{y}\right) F\left(\frac{b(x)-b(x-y)}{y}\right) u(x-y) d y .
$$

Then we have $\|A u\| \leqq C\|F\|_{\mathscr{B}^{1}(\Omega)}\|a\|_{k}\left(1+\|b\|_{2}\right)\|u\|_{s}$ where $C=C(k, s, N)>0, \Omega$ is an open set containing the convex hull of

$$
\left\{\frac{b(x)-b(x-y)}{y} \mid-\infty<x, y<+\infty\right\} \text { and } F \in \mathscr{B}^{1}(\Omega) .
$$

Proof. For a function $f(x)$, we put

$$
\tilde{f}(x, y)=\frac{f(x)-f(x-y)}{y}, \quad f^{\prime}(x)=\frac{d f}{d x}(x) .
$$

It is easily seen that $\partial \tilde{f}(x, y) / \partial y+\tilde{f}^{\prime}(x, y)=-(1 / y)\left(\tilde{f}(x, y)-f^{\prime}(x)\right)$. Using the formula

$$
F(\tilde{b})-F\left(b^{\prime}\right)=\sum_{1}^{N}\left\{\int_{0}^{1} \frac{\partial F}{\partial z_{j}}\left(t \tilde{b}+(1-t) b^{\prime}\right) d t\right\}\left(\tilde{b}_{j}-b_{j}^{\prime}\right)
$$


(we write this in the form $F(\tilde{b})-F\left(b^{\prime}\right)=\sum_{1}^{N} F_{j}\left(\tilde{b}_{j}-b_{j}^{\prime}\right)$ ), we can write $A u$ in the form

$$
\begin{aligned}
& A u=F\left(b^{\prime}\right) \int\left(D_{y}^{k} \frac{a(x)-a(x-y)}{y}\right) u(x-y) d y+\sum_{1}^{N} A_{j} u=B u+\sum_{1}^{N} A_{j} u \\
& A_{j} u=\int\left(D_{y}^{k} \frac{a(x)-a(x-y)}{y}\right) F_{j}\left(\tilde{b}_{j}-b_{j}^{\prime}\right) u(x-y) d y .
\end{aligned}
$$

First we assume that $k=0$. Then we have

$$
A_{j} u=\int a(x) F_{j} \frac{\tilde{b}_{j}-b_{j}^{\prime}}{y} u(x-y) d y+\int a(x-y) F_{j}\left(\frac{\partial}{\partial y} \tilde{b}_{j}+\tilde{b}_{j}^{\prime}\right) u(x-y) d y .
$$

For $0<r<1$,

$$
\begin{aligned}
\left|\frac{\tilde{b}_{j}-b_{j}^{\prime}}{y}\right| & \leqq\left|\frac{1}{y} \int_{-1}^{0}\left(b_{j}^{\prime}(x+t y)-b_{j}^{\prime}(x)\right) d t\right| \\
& \leqq \int_{-1}^{0}\left|b_{j}^{\prime}(x+t y)-b_{j}^{\prime}(x)\right||t y|^{-r}|t|^{r}|y|^{r-1} d t \\
& \leqq C\left\|D b_{j}\right\|_{\mathscr{G} r}(1+|y|)^{-r}|y|^{r-1}
\end{aligned}
$$

By choosing $r, p$ and $q$ such that $0<r<1 / 2,2<p<\infty,(1-r) q<1$ and $(1 / p)+(1 / q)=1$, we obtain from Corollary 2.6

$$
\int(1+|y|)^{-r}|y|^{r-1}|u(x-y)| d y \leqq\left\|(1+|y|)^{-r}|y|^{r-1}\right\|_{L_{q}}\|u\|_{L_{p}} \leqq C\|u\|_{s}
$$

and from Lemma 2.5

$$
\left\|D b_{j}\right\|_{\mathscr{A} r} \leqq C\left\|D b_{j}\right\|_{1} \leqq C\left\|b_{j}\right\|_{2}, \quad \sup |u(x)| \leqq C\|u\|_{s} .
$$

Therefore we have

$$
\begin{aligned}
\left|A_{j} u(x)\right| \leqq & C \sup \left|F_{j}\right|\left\|b_{j}\right\|_{2}\|u\|_{s}|a(x)| \\
& +C \sup \left|F_{j}\right|\|u\|_{s}\left\{\left\{\left|D_{y} \frac{b_{j}(x)-b_{j}(x-y)}{y}\right||a(x-y)|\right.\right. \\
& \left.+\left|\frac{b_{j}^{\prime}(x)-b_{j}^{\prime}(x-y)}{y}\right||a(x-y)|\right\} d y .
\end{aligned}
$$

Using Lemma 4.4, we have $\left\|A_{j} u\right\| \leqq C$ sup $\left|F_{j}\right|\|a\|\left\|b_{j}\right\|_{2}\|u\|_{s^{*}}$. Next we assume that $k>0$. From (4.7) we see that

$$
\partial_{y}^{k} \frac{a(x)-a(x-y)}{y}=-\frac{1}{y}\left\{(-1)^{k} a^{(k)}(x-y)+k \partial_{y}^{k-1} \frac{a(x)-a(x-y)}{y}\right\} .
$$

This and $\tilde{b}_{j}-b_{j}^{\prime}=-y\left(\partial_{y} \tilde{b}_{j}+\tilde{b}_{j}^{\prime}\right)$ show that 


$$
\begin{aligned}
A_{j} u= & (-i)^{k} \\
& \times \int\left\{(-1)^{k} a^{(k)}(x-y)+k \partial_{y}^{k-1} \frac{a(x)-a(x-y)}{y}\right\} F_{j}\left(\partial_{y} \tilde{b}_{j}+\tilde{b}_{j}^{\prime}\right) u(x-y) d y .
\end{aligned}
$$

By Lemmas 4.4 and 4.5, we have

$$
\begin{aligned}
\left\|A_{j} u\right\| \leqq & C \sup \left|F_{j}\right|\left(\left\|b_{j}\right\|_{1+1 / 2}+\left\|b_{j}^{\prime}\right\|_{1 / 2}\right)\left\|a^{(k)}\right\| \sup |u| \\
& +C \sup \left|F_{j}\right|\|a\|_{k-1+1}\left(\left\|b_{j}\right\|_{1+1}+\left\|b_{j}^{\prime}\right\|_{0+1}\right) \sup |u| \\
\leqq & C \sup \left|F_{j}\right|\|a\|_{k}\left\|b_{j}\right\|_{2}\|u\|_{s} .
\end{aligned}
$$

Applying Lemma 4.3 to $B u$ we have for any $k \geqq 0$

$$
\|A u\| \leqq C \sup \left|F\left(b^{\prime}\right)\right|\|a\|_{k}\|u\|_{s}+C \sum_{1}^{N} \sup \left|F_{j}\right|\|a\|_{k}\left\|b_{j}\right\|_{2}\|u\|_{s},
$$

which proves the lemma.

If $F$ has the form $F(z)=z_{1} \cdots z_{M} G\left(z_{M+1}, \ldots, z_{N}\right)$, then

$$
\sup \left|F\left(b^{\prime}\right)\right| \leqq\left(\sup \left|b_{1}^{\prime}\right|\right) \cdots\left(\sup \left|b_{M}^{\prime}\right|\right) \sup |G|
$$

and

$$
\sup \left|F_{j}\right| \leqq \begin{cases}\left(\prod_{1}^{M} \sup \left|b_{i}^{\prime}\right|\right) \sup \left|\frac{\partial G}{\partial z_{j}}\right| & \text { for } \\ \left(\prod_{i \neq j} \sup \left|b_{i}^{\prime}\right|\right) \sup |G| & \text { for } 1 \leqq j \leqq M\end{cases}
$$

Using (4.9) and $\sup \left|b_{j}^{\prime}\right| \leqq C\left\|b_{j}\right\|_{2}$, we have

Lemma 4.10. Let $k$ be an integer $\geqq 0, d=\left(d_{1}, \ldots, d_{N}\right), d_{j}$ be real-valued and

$$
\begin{aligned}
A u(x)=\int & \left(D_{y}^{k} \frac{a(x)-a(x-y)}{y}\right) \\
& \times \prod_{1}^{M} \frac{b_{j}(x)-b_{j}(x-y)}{y} G\left(\frac{d(x)-d(x-y)}{y}\right) u(x-y) d y .
\end{aligned}
$$

Then we have

$$
\|A u\| \leqq C\|G\|_{\mathscr{T}^{1}(\Omega)}\|a\|_{k}\left\|b_{1}\right\|_{2} \cdots\left\|b_{M}\right\|_{2}\left(1+\|d\|_{2}\right)\|u\|_{s},
$$

where $C=C(k, s, M, N)>0, \Omega$ is an open set containing the convex hull of $\{(d(x)-d(x-y)) / y \mid-\infty<x, y<+\infty\}$ and $G \in \mathscr{B}^{1}(\Omega)$.

Lemma 4.11. Let $m$ be an integer $\geqq 2, s>1 / 2, b=\left(b_{1}, \ldots, b_{N}\right), b_{j}$ be realvalued and

$$
A u(x)=\int \frac{a(x)-a(y)}{x-y} F\left(\frac{b(x)-b(y)}{x-y}\right) u(y) d y
$$


Then we have

$$
\|A u\|_{m} \leqq C\|F\|_{\mathscr{Q} m(\Omega)}\|a\|_{m}\left(1+\|b\|_{m}\right)^{m}\|u\|_{s}
$$

where $C=C(m, s, N)>0, \Omega$ is an open set containing the convex hull of $\{(b(x)-b(y)) /(x-y) \mid-\infty<x, y<+\infty\}$ and $F \in \mathscr{B}^{m}(\Omega)$.

Proof. Note that $\|A u\|_{m} \leqq C\left(\|A u\|+\left\|D^{m} A u\right\|\right)$. Putting $k=0$ in Lemma 4.8 and noting that $m \geqq 2$, we have

$$
\|A u\| \leqq C\|F\|_{\mathscr{B}^{m}(\Omega)}\|a\|_{m}\left(1+\|b\|_{m}\right)^{m}\|u\|_{s} .
$$

After the replacement of $y$ by $x-y, m$-times differentiation with respect to $x$ under the integral sign and the partial integration, $D^{m} A u(x)$ can be written in the form

$$
\begin{aligned}
D^{m} A u(x) & =\sum_{0}^{m}\left(\begin{array}{c}
m \\
k
\end{array}\right) \int\left\{D_{y}^{k} D_{x}^{m-k}(\tilde{a} F(\tilde{b}))\right\} u(x-y) d y=\sum_{0}^{m}\left(\begin{array}{c}
m \\
k
\end{array}\right) A_{k} u(x), \\
A_{k} u(x)= & \int\left(D_{y}^{k} D_{x}^{m-k} \tilde{a}\right) F(\tilde{b}) u(x-y) d y \\
& +\sum_{1}^{N} \int \tilde{a} \frac{\partial F}{\partial z_{j}}(\tilde{b})\left(D_{y}^{k} D_{x}^{m-k} \tilde{b}_{j}\right) u(x-y) d y \\
& +\sum_{J, n, p, q} \int\left(D_{y}^{p_{0}} D_{x}^{q_{0}} \tilde{a}\right) F^{J, n, p, q}(\tilde{b}) \prod_{j=1}^{J}\left(D_{y}^{p_{j}} D_{x}^{q_{j}} \tilde{b}_{n_{j}}\right) u(x-y) d y
\end{aligned}
$$

where $J, n, p$ and $q$ move in the set such that

$$
\left\{\begin{array}{l}
1 \leqq J \leqq m \\
p_{j} \leqq k, q_{j} \leqq m-k, p_{j}+q_{j} \leqq m-1, \quad(0 \leqq j \leqq J) \\
1 \leqq n_{j} \leqq N, 1 \leqq p_{j}+q_{j}, \quad(1 \leqq j \leqq J) \\
p_{0}+q_{0}+\cdots+p_{J}+q_{J}=m
\end{array}\right.
$$

and $F^{J, p, q, n}(z)$ is the linear combination of $(\partial / \partial z)^{\alpha} F(z),|\alpha| \leqq J$. We put $A_{k} u$ $=A_{k 1} u+A_{k 2} u+A_{k 3} u$. Since $D_{x}^{m-k} \tilde{a}=\widetilde{D^{m-k} a}$, applying Lemma 4.8 to $A_{k 1} u$, we have

$$
\begin{aligned}
\left\|A_{k 1} u\right\| & \leqq C\|F\|_{\mathscr{F}^{1}(\Omega)}\left\|D^{m-k} a\right\|_{k}\left(1+\|b\|_{2}\right)\|u\|_{s} \\
& \leqq C\|F\|_{\mathscr{G}^{m}(\Omega)}\|a\|_{m}\left(1+\|b\|_{m}\right)^{m}\|u\|_{s} .
\end{aligned}
$$

From Lemma 4.10 we have

$$
\begin{aligned}
\left\|A_{k 2} u\right\| & \leqq \sum_{1}^{N} C\left\|\frac{\partial F}{\partial z_{j}}\right\|_{\mathscr{R}^{1}(\Omega)}\left\|D^{m-k} b_{j}\right\|_{k}\|a\|_{2}\left(1+\|b\|_{2}\right)\|u\|_{s} \\
& \leqq C\|F\|_{\mathscr{B}^{m}(\Omega)}\|a\|_{m}\left(1+\|b\|_{m}\right)^{m}\|u\|_{s} .
\end{aligned}
$$

We may carry out the estimate for $A_{k 3} u$ under the assumption that 


$$
p_{1}+q_{1} \geqq p_{j}+q_{j}, \quad 1 \leqq j \leqq J .
$$

When $p_{0}+q_{0}+p_{1}+q_{1} \geqq 2$, we see from (4.12) that $p_{j}+q_{j} \leqq m-2$ for $2 \leqq j \leqq J$. Using (4.7), we have for $2 \leqq j \leqq J$

$$
\left|D_{y}^{p_{j}} D_{x}^{q_{j}} \tilde{b}_{n_{j}}\right| \leqq \sup \left|D^{1+p_{j}+q_{j}} b_{n_{j}}\right| \leqq C\left\|b_{n_{j}}\right\|_{1+p_{j}+q_{j}+1} \leqq C\left\|b_{n_{j}}\right\|_{m} .
$$

Hence

$$
\begin{aligned}
I & =\left|\left(D_{y}^{p_{0}} D_{x}^{q_{0}} \tilde{a}\right) \prod_{j=1}^{J}\left(D_{y}^{p_{j}} D_{x}^{q_{j}} \tilde{b}_{n_{j}}\right) F^{J, n, p, q}(\tilde{b})\right| \\
& \leqq C\left|D_{y}^{p_{0}} D_{x}^{q_{0}} \tilde{a}\right|\left|D_{y}^{p_{1}} D_{x}^{q_{1}} \tilde{b}_{n_{1}}\right|\left\|b_{n_{2}}\right\|_{m} \cdots\left\|b_{n_{J}}\right\|_{m} \sup \left|F^{J, n, p, q}(\tilde{b})\right| .
\end{aligned}
$$

When $p_{0}+q_{0}+p_{1}+q_{1}<2$, by (4.13) and $p_{j}+q_{j} \geqq 1$ for $j \geqq 1$ we have $p_{0}+q_{0}=0$, $p_{j}+q_{j}=1$ for $j \geqq 1$. Moreover from (4.12) it follows that $J=m$. If $m \geqq 3$ then

$$
\left|D_{y}^{p_{j}} D_{x}^{q_{j}} \tilde{b}_{n_{j}}\right| \leqq C\left\|b_{n_{j}}\right\|_{3} \leqq C\left\|b_{n_{j}}\right\|_{m} \quad \text { for } \quad j \geqq 2 .
$$

If $m=2$ then $J=m=2$ and

$$
\begin{aligned}
I & \leqq C(\sup |\tilde{a}|)\left|D_{y}^{p_{1}} D_{x}^{q_{1}} \tilde{b}_{n_{1}}\right|\left|D_{y}^{p_{2}} D_{x}^{q_{2}} \tilde{b}_{n_{2}}\right| \sup \left|F^{J, n, p, q}(\tilde{b})\right| \\
& \leqq C\left|D_{y}^{p_{1}} D_{x}^{q_{1}} \tilde{b}_{n_{1}}\right|\left|D_{y}^{p_{2}} D_{x}^{q_{2}} \tilde{b}_{n_{2}}\right|\|a\|_{2} \sup \left|F^{J, n, p, q}(\tilde{b})\right|
\end{aligned}
$$

By Lemma 4.5 we have

$$
\left\|A_{k 3} u\right\| \leqq C\|F\|_{\mathscr{B}^{m}(\Omega)}\|a\|_{m}\left(1+\|b\|_{m}\right)^{m}\|u\|_{s} .
$$

This completes the proof.

The same consideration as in the derivation of Lemma 4.10 from (4.9) leads to the following lemma.

Lemma 4.14. Let $m$ be an integer $\geqq 2, s>1 / 2, b=\left(b_{1}, \ldots, b_{N}\right), b_{j}$ be realvalued and

$$
A u(x)=\int\left(\prod_{j=1}^{M} \frac{a_{j}(x)-a_{j}(y)}{x-y}\right) F\left(\frac{b(x)-b(y)}{x-y}\right) u(y) d y .
$$

Then we have

$$
\|A u\|_{m} \leqq C\|F\|_{\mathscr{B}(\Omega)}\left(\prod_{1}^{M}\left\|a_{j}\right\|_{m}\right)\left(1+\|b\|_{m}\right)^{m}\|u\|_{s}
$$

where $C=C(m, s, M, N)>0, \Omega$ is an open set containing the convex hull of $\{(b(x)-b(y)) /(x-y) \mid-\infty<x, y<+\infty\}$ and $F \in \mathscr{B}^{m}(\Omega)$.

Lemma 4.15. Let $m$ be an integer $\geqq 2, s>1 / 2$ and $A=A(a, b)$ be the operator defined in the above lemma. Then we have 


$$
\begin{aligned}
& \left\|A\left(a^{1}, b^{1}\right) u-A\left(a^{2}, b^{2}\right) u\right\|_{m} \\
& \begin{aligned}
\leqq C\|F\|_{\mathscr{B}^{m+1}(\Omega)}\left(1+\left\|a^{1}\right\|_{m}\right. & \left.+\left\|a^{2}\right\|_{m}\right)^{M}\left(1+\left\|b^{1}\right\|_{m}\right. \\
& \left.+\left\|b^{2}\right\|_{m}\right)^{m}\left(\left\|a^{1}-a^{2}\right\|_{m}+\left\|b^{1}-b^{2}\right\|_{m}\right)\|u\|_{s}
\end{aligned}
\end{aligned}
$$

where $C=C(m, s, M, N)>0, \Omega$ is an open set containing the convex hull of $\left\{\left(b^{k}(x)-b^{k}(y)\right) /(x-y) \mid-\infty<x, y<+\infty, k=1,2\right\}$ and $F \in \mathscr{B}^{m+1}(\Omega)$.

Proof. Note that

$$
\begin{aligned}
A\left(a^{1}, b^{1}\right) u-A\left(a^{2}, b^{2}\right) u & =\int_{0}^{1} \frac{\partial}{\partial t} A\left(t a^{1}+(1-t) a^{2}, t b^{1}+(1-t) b^{2}\right) u d t \\
& =\int_{0}^{1} d t \int G u d y
\end{aligned}
$$

where

$$
\begin{aligned}
G= & \sum_{k=1}^{M}\left(\check{a}_{k}^{1}-\check{a}_{k}^{2}\right) \prod_{j \neq k}\left\{t \check{a}_{j}^{1}+(1-t) \check{a}_{j}^{2}\right\} F\left(t \check{b}^{1}+(1-t) \check{b}^{2}\right) \\
& +\prod_{j=1}^{M}\left\{t \check{a}_{j}^{1}+(1-t) \check{a}_{j}^{2}\right\} \sum_{k=1}^{N} \frac{\partial F}{\partial z_{k}}\left(t \check{b}^{1}+(1-t) \check{b}^{2}\right)\left(\check{b}_{k}^{1}-\check{b}_{k}^{2}\right), \\
\check{a}= & \frac{a(x)-a(y)}{x-y}, \quad \check{b}=\frac{b(x)-b(y)}{x-y} .
\end{aligned}
$$

Since

$$
\begin{aligned}
\left\|A\left(a^{1}, b^{1}\right) u-A\left(a^{2}, b^{2}\right) u\right\|_{m} & =\left\|\int_{0}^{1} d t \int G u d y\right\|_{m} \\
& \leqq \int_{0}^{1} d t\left\|\int G u d y\right\|_{m}
\end{aligned}
$$

the required estimate is obtained from Lemma 4.14.

Remark 4.17. The above two lemmas hold also for $m+r, m \geqq 2,0<r<1$. If we define the translation operator $T_{z}, z \in \mathbb{R}^{1}$, by $T_{z} f(x)=f(x+z)$ then it is clear that $T_{z} A(a, b) u=A\left(T_{z} a, T_{z} b\right) T_{z} u$. Since $T_{z} A(a, b) u-A(a, b) u=$ $A\left(T_{z} a, T_{z} b\right) T_{z} u-A(a, b) T_{z} u+A(a, b)\left(T_{z} u-u\right)$, by Remark 2.8, Lemma 4.14 and (4.16) we have

$$
\|A u\|_{m+r} \leqq C\|F\|_{\mathscr{B}^{m+1}(\Omega)} \prod_{1}^{M}\left\|a_{j}\right\|_{m+r}\left(1+\|b\|_{m+r}\right)^{m+1}\|u\|_{s+r} .
$$

This combined with (4.16) leads to the estimate

$$
\begin{aligned}
& \left\|A\left(a^{1}, b^{1}\right) u-A\left(a^{2}, b^{2}\right) u\right\|_{m+r} \\
& \leqq C\|F\|_{\mathscr{R}^{m+2}(\Omega)}\left(1+\left\|a^{1}\right\|_{m+r}+\left\|a^{2}\right\|_{m+r}\right)^{M}\left(1+\left\|b^{1}\right\|_{m+r}+\left\|b^{2}\right\|_{m+r}\right)^{m+1} \\
& \times\left(\left\|a^{1}-a^{2}\right\|_{m+r}+\left\|b^{1}-b^{2}\right\|_{m+r}\right)\|u\|_{s+r} .
\end{aligned}
$$




\subsection{Operators of the Form (4.2).}

Lemma 4.18. Let $m$ be an integer $\geqq 1, h>0$ and

$$
A u(x)=\int\left(\prod_{1}^{M} \frac{a_{j}(x)-b_{j}(y)}{x-y \pm i h}\right) F\left(\frac{f(x)-g(y)}{x-y \pm i h}\right) u(y) d y .
$$

Then

$$
\|A u\|_{m} \leqq C\|F\|_{\mathscr{B}^{m}(\Omega)} \prod_{1}^{M}\left(\left\|a_{j}\right\|_{m}+\left\|b_{j}\right\|_{1}\right)\left(1+\|f\|_{m}+\|g\|_{1}\right)^{m}\|u\|
$$

where $C=C(m, h, M, N)>0, \Omega$ is an open set containing

$$
\left\{\frac{f(x)-g(y)}{x-y \pm i h} \mid-\infty<x, y<+\infty\right\} \text { and } F \in \mathscr{B}^{m}(\Omega) .
$$

Proof. Put

$$
I(x)=\int\left|\frac{u(y) v(y)}{x-y \pm i h}\right| d y .
$$

If $v=1$ then $I(x) \leqq\left\|(x \pm i h)^{-1}\right\|\|u\| \leqq C\|u\|$. Since

$$
I(x)^{2} \leqq\|u\|^{2} \int\left|\frac{v(y)}{x-y \pm i h}\right|^{2} d y,
$$

by Hausdorff-Young's inequality, we have

$$
\|I\|^{2} \leqq\|u\|^{2}\left\|\frac{1}{|x \pm i h|^{2}}\right\|_{L_{1}}\left\||v|^{2}\right\|_{L_{1}} \text {, i.e., } \quad\|I\| \leqq C\|u\|\|v\| .
$$

From

$$
|A u(x)| \leqq\left(\left|a_{1}(x)\right| \int\left|\frac{u(y)}{x-y \pm i h}\right| d y+\int\left|\frac{b_{1}(y) u(y)}{x-y \pm i h}\right| d y\right) \sup _{x, y}\left|\prod_{2}^{M} \frac{a_{j}(x)-b_{j}(y)}{x-y \pm i h} F\right|
$$

we obtain

$$
\|A u\| \leqq C\left(\left\|a_{1}\right\|\|u\|+\left\|b_{1}\right\|\|u\|\right)\left\{\prod_{2}^{M} \frac{1}{h}\left(\left\|a_{j}\right\|_{1}+\left\|b_{j}\right\|_{1}\right)\right\}\|F\|_{\mathscr{B}^{0}(\Omega)} .
$$

By the differentiation under the integral sign we can divide $D^{m} A u$ into two parts: The first contains $D^{m} a, D^{m} f$ and the second $D^{k} a, D^{k} f, k<m$. The above method is available for the first part and also for the second if we note that $\mid D_{x}^{j}(x-y$ $\pm i h)^{-1}|\leqq C| x-y \pm\left. i h\right|^{-1}$. Since $\|A u\|_{m} \leqq C\|A u\|+C\left\|D^{m} A u\right\|$, we obtain the required inequality.

Lemma 4.19. Let $m$ be an integer $\geqq 1, h>0$ and $A=A(a, b, f, g)$ be the operator defined in the above lemma. Then we have 


$$
\begin{aligned}
\| A\left(a^{1},\right. & \left.b^{1}, f^{1}, g^{1}\right) u-A\left(a^{2}, b^{2}, f^{2}, g^{2}\right) u \|_{m} \\
\leqq & C\|F\|_{\mathscr{g}^{m+1}(\Omega)}\left(1+\left\|a^{1}\right\|_{m}+\left\|a^{2}\right\|_{m}+\left\|b^{1}\right\|_{1}+\left\|b^{2}\right\|_{1}\right)^{M} \\
& \times\left(1+\left\|f^{1}\right\|_{m}+\left\|f^{2}\right\|_{m}+\left\|g^{1}\right\|_{1}+\left\|g^{2}\right\|_{1}\right)^{m} \\
& \times\left(\left\|a^{1}-a^{2}\right\|_{m}+\left\|b^{1}-b^{2}\right\|_{1}+\left\|f^{1}-f^{2}\right\|_{m}+\left\|g^{1}-g^{2}\right\|_{1}\right)\|u\|
\end{aligned}
$$

where $C=C(m, h, M, N)>0, \Omega$ is an open set containing the convex hull of

$$
\left\{\frac{f^{k}(x)-g^{k}(y)}{x-y \pm i h} \mid-\infty<x, y<+\infty, k=1,2\right\} \text { and } F \in \mathscr{B}^{m+1}(\Omega) .
$$

The lemma is proved by the method used for Lemma 4.15 .

Remark 4.20. By the same consideration as in Remark 4.17, we have for $m \geqq 1,0<r<1$,

$$
\begin{aligned}
&\|A u\|_{m+r} \leqq C\|F\|_{\mathscr{g}^{m+1}(\Omega)} \prod_{1}^{M}\left(\left\|a_{j}\right\|_{m+r}+\left\|b_{j}\right\|_{1+r}\right)\left(1+\|f\|_{m+r}+\|g\|_{1+r}\right)^{m+1}\|u\|_{r} \\
&\left\|A\left(a^{1}, b^{1}, f^{1}, g^{1}\right) u-A\left(a^{2}, b^{2}, f^{2}, g^{2}\right) u\right\|_{m+r} \\
& \leqq C\|F\|_{\mathscr{g}^{m+2}(\Omega)}\left(1+\left\|a^{1}\right\|_{m+r}+\left\|a^{2}\right\|_{m+r}+\left\|b^{1}\right\|_{1+r}+\left\|b^{2}\right\|_{1+r}\right)^{M} \\
& \times\left(1+\left\|f^{1}\right\|_{m+r}+\left\|f^{2}\right\|_{m+r}+\left\|g^{1}\right\|_{1+r}+\left\|g^{2}\right\|_{1+r}\right)^{m+1}\left(\left\|a^{1}-a^{2}\right\|_{m+r}\right. \\
&\left.+\left\|b^{1}-b^{2}\right\|_{1+r}+\left\|f^{1}-f^{2}\right\|_{m+r}+\left\|g^{1}-g^{2}\right\|_{1+r}\right)\|u\|_{r}
\end{aligned}
$$

4.3. The Operator $\boldsymbol{K}$. Let $A$ be an operator of the form (4.1) or (4.2) which we write in the form $A u(x)=\int A(x, y) u(y) d y$. Since

$$
\begin{aligned}
& {\left[\frac{\partial}{\partial x}, A\right] u=\int\left\{\left(\frac{\partial}{\partial x}+\frac{\partial}{\partial y}\right) A(x, y)\right\} u(y) d y} \\
& \left(\frac{\partial}{\partial x}+\frac{\partial}{\partial y}\right) \frac{a(x)-a(y)}{x-y}=\frac{a^{\prime}(x)-a^{\prime}(y)}{x-y}, \quad a^{\prime}(x)=\partial_{x} a(x) \\
& \left(\frac{\partial}{\partial x}+\frac{\partial}{\partial y}\right) \frac{a(x)-b(y)}{x-y \pm i h}=\frac{a^{\prime}(x)-b^{\prime}(y)}{x-y \pm i h},
\end{aligned}
$$

we see that $[\partial / \partial x, A]$ is the sum of operators of the form (4.1) or (4.2). If $A(x, y)$ and $u$ depend on $t$, then $[\partial / \partial t, A] u=\int(\partial A(x, y) / \partial t) u(y) d y$. Hence $[\partial / \partial t, A]$ is the linear combination of operators of the form (4.1) or (4.2).

Note that $\log (1+z)=z f(z)$ where $f(z)$ is holomorphic in $z, \operatorname{Re} z>-1$ and

$$
\begin{aligned}
& \left|\frac{a(x)-a(y)}{x-y}\right| \leqq \sup \left|a^{\prime}(x)\right| \leqq C\|a\|_{1+s}, \\
& \left|\frac{a(x)-b(y)}{x-y \pm i h}\right| \leqq \frac{1}{h}(|a(x)|+|b(y)|) \leqq C\left(\|a\|_{s}+\|b\|_{s}\right), \quad s>\frac{1}{2} .
\end{aligned}
$$

By (3.5) and (3.7) we see that, if $X$ and $b$ are small in $H^{s}$, then $A_{j}$ can be written 
in the form (4.1) or (4.2) where $u$ is replaced by $d u / d y$. This situation leads us to the following

Definition 4.21. Let $0 \leqq r \leqq s, 0 \leqq t \leqq s . ~ L(r, s ; t)$ is the totality of $M$ satisfying the following conditions: 1) $M=M(P ; P(J))$ is the linear operator depending on $P=\left(P_{1}, \ldots, P_{k}\right)$ where $P_{j}$ is the real-valued function, $J$ is the subset of $\{1, \ldots, k\}, P(J)=\left(P_{j_{1}}, \ldots, P_{j_{l}}\right)$ if $J=\left\{j_{1}, \ldots, j_{l}\right\}$ and we write $M=M(P ; 0)$ if $J$ is empty. 2) There exists $d=d(M, t)>0$ such that if $d_{0}>0, P, P^{0} \in H^{s},\|P\|_{s}$, $\left\|P^{0}\right\|_{s} \leqq d_{0},\|P(J)\|_{t},\left\|P^{0}(J)\right\|_{t} \leqq d$ then $\|M(P ; P(J)) u\|_{s} \leqq C\|u\|_{r}, \| M(P ; P(J)) u$ $-M\left(P^{0} ; P^{0}(J)\right) u\left\|_{s} \leqq C\right\| P-P^{0}\left\|_{s}\right\| u \|_{r}$ for $u \in H^{r}$ where $C=C\left(r, s, t, d, d_{0}\right)>0$. $L_{0}(r, s ; t)$ consists of $M \in L(r, s ; t)$ such that $\|M(P ; P(J)) u\|_{s} \leqq C\|P\|_{s}\|u\|_{r}$.

\section{Lemma 4.22.}

1) $L(r, s ; t), L_{0}(r, s ; t)$ are algebras.

2) $L_{0}(r, s ; t)$ is a two-sided $L(r, s ; t)$-module.

3) If $f$ is smooth in a neighbourhood of $0 \in \mathbb{R}^{k}$ then the operator $M$ defined by $M(P ; P) u=f(P) u$ belongs to $L(s, s ; t)$ for $1 / 2<t \leqq s, 1 \leqq s$.

4) $M(P ; P)=\left(1+P_{1}+P_{2} i \operatorname{sgn} D\right)^{-1} \in L(s, s ; t)$ for $1 / 2<t \leqq s$.

Proof. 1) and 2) are trivial. 3) follows from Lemma 2.11 and Remark 2.12. It remains to show 4). Let $P_{1}, P_{2} \in H^{t}$. Then by Remark 2.10, $\left\|\left(P_{1}+P_{2} i \operatorname{sgn} D\right) u\right\|_{t} \leqq C\|P\|_{t}\|u\|_{t}$. Hence if $P_{1}$ and $P_{2}$ are small in $H^{t}$, then we have $(a)\|M(P ; P) u\|_{t} \leqq C\|u\|_{t}$. Note that $T_{z} u(x)=u(x+z)=\exp (i z D) u(x)$ and $\left[T_{z}, P_{j}\right]=\left(P_{j}(\cdot+z)-P_{j}\right) T_{z}$. Since $T_{z} M u-M u=\left[T_{z}, M\right] u+M\left(T_{z} u-u\right)$ $=-M\left[T_{z}, M^{-1}\right] M u+M\left(T_{z} u-u\right)$ and

$$
\left[T_{z}, 1+P_{1}+P_{2} i \operatorname{sgn} D\right]=\left(P_{1}(\cdot+z)-P_{1}\right) T_{z}+\left(P_{2}(\cdot+z)-P_{2}\right) T_{z} i \operatorname{sgn} D,
$$

we have (b) $\left\|T_{z} M u-M u\right\|_{t} \leqq C\left\|T_{z} P-P\right\|_{t}\|u\|_{t}+C\left\|T_{z} u-u\right\|_{t}$. From Remark 2.8 it follows that (c) $\|M u\|_{t+r} \leqq C\|u\|_{t+r}$ if $0<r<1, P_{1}, P_{2} \in H^{t+r}$. Using (c) in place of $(a)$ we have $(b)$ with $t+r$, which leads to $(c)$ with $t+2 r$. Repeating this procedure, we have $\|M(P ; P) u\|_{s} \leqq C\|u\|_{s}$. This, combined with $M-M_{0}$ $=-M\left(M^{-1}-M_{0}^{-1}\right) M_{0}, M=M(P ; P) \quad$ and $M_{0}=M\left(P^{0} ; P^{0}\right)$, shows that $\left\|M(P ; P) u-M\left(P^{0} ; P^{0}\right) u\right\|_{s} \leqq C\left\|P-P^{0}\right\|_{s}\|u\|_{s}$. The proof is complete.

By the facts stated in the beginning of this article and Lemmas 4.14, 4.15, 4.17-4.20 we have

Lemma 4.23. Let $m$ be an integer $\geqq 2,0 \leqq r<1$ and $1 / 2<s, t \leqq 1$. Then for the operators $A_{j}$ defined by (3.5) and (3.7), 


$$
\begin{aligned}
& A_{j}\left(X ; X_{1}\right) \in L_{0}(1+s+r, m+r ; 1+t), \quad j=1,2 \\
& A_{j}(X, b ; X, b) \in L_{0}(1+r, m-1+r ; t), \quad j=3,4,7,8 \\
& A_{j}(b ; 0) \in L_{0}(1+s+r, m+r ; 0), \quad j=5,6 .
\end{aligned}
$$

Lemma 4.24. Let $m$ be an integer $\geqq 3$ and $0 \leqq r<1$. Then

$$
K_{1}=K_{1}(X, b ; X, b) \in L_{0}(2+r, m+r ; 3) .
$$

Proof. By the above lemma we see that

$$
\begin{aligned}
& B_{3}(X, b ; X, b)=-i \operatorname{sgn} D e^{-h|D|} b_{x}+A_{3}-A_{4} b_{x} \in L_{0}(1+r, m+r ; 1), \\
& B_{4}\left(b, b_{x} ; 0\right)=i \operatorname{sgn} D b_{x}+A_{5}-A_{6} b_{x} \in L_{0}(2,2 ; 0) .
\end{aligned}
$$

Therefore

$$
\begin{aligned}
& M_{1}\left(b, b_{x} ; b, b_{x}\right)=\left(1+B_{4}\right)^{-1} \in L(2,2 ; 2), \\
& M_{2}\left(b, b_{x} ; b, b_{x}\right)=B_{4}\left(1+B_{4}\right)^{-1} \in L_{0}(2,2 ; 2) .
\end{aligned}
$$

From (3.9) it follows that

$$
\begin{aligned}
& B_{j}(X, b ; X, b) \in L_{0}(2+r, m+r ; 3), \quad j=1,2, \\
& B_{1}\left(X, b, b_{x} ; X, b, b_{x}\right) \in L_{0}(2,2 ; 2) .
\end{aligned}
$$

Hence $M_{3}\left(X, b, b_{x} ; X, b, b_{x}\right)=\left(1+B_{1}\right)^{-1} \in L(2,2 ; 2)$. In the same way as in the proof of Lemma 4.22 we see that $M_{3} \in L(2+r, 2+r ; 2)$. Consequently we see from (3.8) that $K_{1} \in L_{0}(2+r, m+r ; 3)$. The proof is complete.

Remark 4.25. It is easily seen from the above proof that

$$
K_{1}\left(X, b, b_{x} ; X, b, b_{x}\right) \in L_{0}(2+r, 2+r ; 2), \quad 0 \leqq r<1 .
$$

Assuming that $X$ depends also on $t$, we define

$$
A_{j, k, l}\left(X, \ldots, \partial_{t}^{k} \partial_{x}^{l} X, b, \ldots, \partial_{x}^{l} b ; X, b\right), \quad 1 \leqq j \leqq 8,0 \leqq k, l,
$$

(by $X, \ldots, \partial_{t}^{k} \partial_{x}^{l} X$ we denote the derivatives $\partial_{t}^{p} \partial_{x}^{q} X, p \leqq k, q \leqq l$ ), inductively by $A_{j, 0,0}=A_{j}, A_{j, 0, l}=\left[\frac{\partial}{\partial x}, A_{j, 0, l-1}\right], l \geqq 1, \quad A_{j, k, l}=\left[\frac{\partial}{\partial t}, A_{j, k-1, l}\right], k \geqq 1, l \geqq 0$.

We have seen that $A_{j, k, l}$ can be written in the form of the linear combination of operators of the type (4.1) or (4.2). After this is done, there is no necessity to regard $\partial_{t}^{p} \partial_{x}^{q} X$ in $A_{j, k, l}$ as the derivatives of $X$. Hence we replace $\partial_{t}^{p} \partial_{x}^{q} X$ by $X^{p q}$. By the results of the preceding two articles we have 
Lemma 4.26. Let $m$ be an integer $\geqq 2$ and $0 \leqq r<1$. Then

$$
A_{j, k, l}\left(X^{00}, \ldots, X^{k l}, b, \ldots, \partial_{x}^{l} b ; X^{00}, b\right) \in L_{0}(2+r, m+r ; 2) \text {. }
$$

Since $K_{1}$ is rational in $A_{j}$ and $[R, S T]=[R, S] T+S[R, T],\left[R,(1+T)^{-1}\right]$ $=(1+T)^{-1}[T, R](1+T)^{-1}$ hold for operators $R, S$ and $T$, we define

$$
K_{1 . k, l}\left(X, \ldots, \partial_{t}^{k} \partial_{x}^{l} X, b, \ldots, \partial_{x}^{l} b ; X, b\right)
$$

by the formula used for the definition of $A_{j, k . l}$ and replace $\partial_{t}^{p} \partial_{x}^{q} X$ in $K_{1}$ by $X^{p q}$. By the same consideration as in the proof of Lemma 4.24 we have

Lemma 4.27. Let $m$ be an integer $\geqq 3$ and $0 \leqq r<1$. Then

$$
K_{1, k, l}\left(X^{00}, \ldots, X^{k l}, b, \ldots, \partial_{x}^{l} b ; X^{00}, b\right) \in L_{0}(2+r, m+r ; 3) .
$$

Corollary 4.28. For any integer $m \geqq 3$,

$$
M(X, Z, b ; X, Z, b)=\left\{1+Z_{1}+Z_{2} K(X, b ; X, b)\right\}^{-1} \in L(m, m ; 3) .
$$

Proof. Since $K(X, b ; X, b)=-i \tanh (h D)+K_{1}(X, b ; X, b) \in L(3,3 ; 3)$ we have $M_{1}(X, Z, b ; X, b)=Z_{1}+Z_{2} K(X, b ; X, b) \in L_{0}(3,3 ; 3)$. Hence $M$ $=\left(1+M_{1}\right)^{-1} \in L(3,3 ; 3)$. Since

$$
\left[\frac{\partial}{\partial x}, M\right]=-M\left[\frac{\partial}{\partial x}, M^{-1}\right] M=-M\left\{Z_{1 x}+Z_{2 x} K+Z_{2}\left[\frac{\partial}{\partial x}, K_{1}\right]\right\} M,
$$

we have by the above lemma

$$
\begin{aligned}
\|M u\|_{4} & \leqq C\|M u\|_{3}+C\left\|\frac{\partial}{\partial x} M u\right\|_{3} \\
& \leqq C\|M u\|_{3}+C\left\|\left[\frac{\partial}{\partial x}, M\right] u+M \frac{\partial}{\partial x} u\right\|_{3} \leqq C\|u\|_{4}
\end{aligned}
$$

if $X, Z, b$ are in $H^{4}$ and small in $H^{3}$. In the same way as in the proof of Lemma 4.22 we see that $M \in L(m, m ; 3)$.

\section{§5. Reduction to the Quasilinear System}

We shall reduce the system (1.6) to the quasilinear system such that the unique solvability of the initial value problem for this system assures one for that system and the successive approximation is available for this system. For the usual procedure for the reduction to the quasilinear system, see [9], Chapter I, Section 7.2, Chapter V, Section 1.7 and [10]. 
5.1. Quasilinearization. In quasilinearizing the system

$$
\left(1+X_{1 x}\right) X_{1 t t}+X_{2 x}\left(1+X_{2 t t}\right)=0, \quad X_{2 t}=K X_{1 t},
$$

we use the commutators in order to single out the principal parts of operators. Put $F_{j k}=\left[\partial_{t}^{j} \partial_{x}^{k}, K\right] X_{1 t}$. Since $P^{n} Q=Q P^{n}+\sum_{1}^{n}\left(\begin{array}{c}n \\ j\end{array}\right)[P, Q]_{j} P^{n-j}$ holds for operators $P$ and $Q$ where $[P, Q]_{1}=[P, Q]$ and $[P, Q]_{k+1}=\left[P,[P, Q]_{k}\right], k \geqq 1$, we have

$$
\begin{aligned}
\partial_{t}^{j} \partial_{x}^{k} K= & K \partial_{t}^{j} \partial_{x}^{h}+\sum_{1}^{j}\left(\begin{array}{c}
j \\
p
\end{array}\right) K_{1, p, 0} \partial_{t}^{j-p} \partial_{x}^{k}+\sum_{1}^{k}\left(\begin{array}{c}
k \\
q
\end{array}\right) K_{1,0, q} \partial_{t}^{j} \partial_{x}^{k-q} \\
& +\sum_{1}^{k}\left(\begin{array}{c}
k \\
q
\end{array}\right) \sum_{1}^{j}\left(\begin{array}{c}
j \\
p
\end{array}\right) K_{1, p, q} \hat{\partial}_{t}^{j-p} \partial_{x}^{k-q} .
\end{aligned}
$$

In virtue of Lemma 4.27 the operator $K_{1, p, q} \partial_{x}^{k-q}$ is of order 0 if all functions contained in the kernels are in $H^{m}$ for the sufficiently large $m$, therefore we use the notations

$$
F_{j 0}=F_{j 0}\left(X, \ldots, \partial_{t}^{j} X\right), F_{j k}=F_{j k}\left(X, \ldots, \partial_{t}^{j} \partial_{x}^{k} X, \partial_{t}^{j+1} X_{1}\right), \quad j \geqq 0, k \geqq 1 .
$$

In the precise form,

$$
\left\{\begin{aligned}
F_{j 0}\left(X^{00}, \ldots, X^{j 0}\right)=\sum_{1}^{j}\left(\begin{array}{c}
j \\
p
\end{array}\right) K_{1, p, 0}\left(X^{00}, \ldots, X^{p 0}\right) X_{1}^{j-p+1,0} \\
F_{j k}\left(X^{00}, \ldots, X^{j k}, X_{1}^{j+1.0}\right)=\sum_{1}^{j}\left(\begin{array}{c}
j \\
p
\end{array}\right) K_{1, p, 0}\left(X^{00}, \ldots, X^{p 0}\right) \partial_{x}^{k} X_{1}^{j-p+1,0} \\
+\sum_{1}^{k}\left(\begin{array}{c}
k \\
q
\end{array}\right) K_{1 . n, q}\left(X^{00}, \ldots, X^{0 q}\right) \partial_{x}^{k-q} X_{1}^{j+1,0} \\
+\sum_{1}^{k}\left(\begin{array}{c}
k \\
q
\end{array}\right) \sum_{1}^{j}\left(\begin{array}{c}
j \\
p
\end{array}\right) K_{1, p, q}\left(X^{00}, \ldots, X^{p q}\right) \partial_{x}^{k-q} X_{1}^{j-p+1,0}
\end{aligned}\right.
$$

where in $K_{1, k, l}$ we omit $b, \ldots, \partial_{x}^{l} b ; X^{00}, b$. From (5.1) we obtain

$$
\partial_{t}^{j} \partial_{x}^{k} X_{2 t}=K \partial_{t}^{j} \partial_{x}^{k} X_{1 t}+F_{j h} .
$$

Put

$$
Y=X_{t t}, Z=X_{x}, W=(X, Y, Z), W^{\prime}=\left(X, Y_{1}\right) .
$$

From (5.1) it follows that

$$
\frac{\partial}{\partial t}\left\{\left(1+Z_{1}\right) Y_{1}+Z_{2}\left(1+Y_{2}\right)\right\}=Y_{1} Z_{1 t}+\left(1+Y_{2}\right) Z_{2 t}+\left(1+Z_{1}\right) Y_{1 t}+Z_{2} Y_{2 t}=0
$$

From (5.3) and (3.8) we obtain

$$
\begin{aligned}
X_{2 t x} & =K X_{1 t x}+F_{01}\left(X, X_{x}, X_{1 t}\right) \\
& =-i \operatorname{sgn} D X_{1 t x}+i(\operatorname{sgn} D-\tanh (h D)) \frac{\partial}{\partial x} X_{1 t}+K_{1} \frac{\partial}{\partial x} X_{1 t}+F_{01} .
\end{aligned}
$$


This yields the equation

$$
Z_{2 t}=-i \operatorname{sgn} D Z_{1 t}+F_{010}\left(X, Z, X_{1 t}\right) \text {. }
$$

By the elimination of $Z_{2 t}$ from this and (5.5), we have

$$
Z_{1 t}=-\left\{\left(1+Y_{2}\right)(-i \operatorname{sgn} D)+Y_{1}\right\}^{-1}\left\{\left(1+Y_{2}\right) F_{010}+\left(1+Z_{1}\right) Y_{1 t}+Z_{2} Y_{2 t}\right\}
$$

In virtue of (5.3) with $j=2, k=0$ we have $Y_{2 t}=K(X) Y_{1 t}+F_{20}\left(X, X_{t}, Y\right)$ $=f_{2}\left(W, W_{t}^{\prime}\right)$, therefore the substitution of $Y_{2 t}$ in (5.7) by $f_{2}$ and $Z_{1 t}$ in (5.6) by the right-hand side of (5.7) lead to the equations

$$
Y_{2 t}=f_{2}\left(W, W_{t}^{\prime}\right), Z_{1 t}=f_{3}\left(W, W_{t}^{\prime}\right), Z_{2 t}=f_{4}\left(W, W_{t}^{\prime}\right)
$$

Remark 5.9. If functions $X, Y, Z$ satisfy the equations (5.8), then

$$
\left\{\begin{array}{l}
Z_{2 t}=-i \operatorname{sgn} D Z_{1 t}+F_{010}\left(X, Z, X_{1 t}\right) \\
\frac{\partial}{\partial t}\left\{\left(1+Z_{1}\right) Y_{1}+Z_{2}\left(1+Y_{2}\right)\right\}=0
\end{array}\right.
$$

Let us now proceed to the equation for $Y_{1}$. From (5.1) we have $(\partial / \partial t)^{2}$. $\left\{\left(1+X_{1 x}\right) Y_{1}+X_{2 x}\left(1+Y_{2}\right)\right\}=0$. Replacing $X_{x t t}$ by $Y_{x}$ and $X_{x t}$ by $Z_{t}$, we obtain $\left(1+Z_{1}\right) Y_{1 t t}+Z_{2} Y_{2 t t}+Y_{1} Y_{1 x}+\left(1+Y_{2}\right) Y_{2 x}+2 Y_{t} \cdot Z_{t}=0$. By (5.3) with $j=3, k=0$ we have $Y_{2 t t}=K(X) Y_{1 t t}+F_{30}\left(X, X_{t}, Y, Y_{t}\right)$. Eliminating $Y_{2 t t}$ from these equations, we obtain

$$
Y_{1 t t}=-\left(1+Z_{1}+Z_{2} K\right)^{-1}\left(Y_{1} Y_{1 x}+\left(1+Y_{2}\right) Y_{2 x}+Z_{2} F_{30}+2 Y_{t} \cdot Z_{t}\right) .
$$

By (5.3) with $j=k=1$, we have $Y_{2 x}=K(X) Y_{1 x}+F_{11}\left(X, X_{t}, Z, Z_{t}, Y_{1}\right)$. Hence the above two equations yield

$$
\begin{aligned}
Y_{1 t t}= & -\left(1+Z_{1}+Z_{2} K\right)^{-1}\left\{Y_{1}+\left(1+Y_{2}\right) K\right\} Y_{1 x} \\
& -\left(1+Z_{1}+Z_{2} K\right)^{-1}\left\{\left(1+Y_{2}\right) F_{11}+Z_{2} F_{30}+2 Y_{t} \cdot Z_{t}\right\}
\end{aligned}
$$

Using the identity

$$
\begin{aligned}
& \left(1+Z_{1}-Z_{2} K\right)\left(1+Z_{1}+Z_{2} K\right) \\
& \quad=\left(1+Z_{1}\right)^{2}+Z_{2}^{2}-Z_{2}\left\{\left[K, Z_{1}\right]+\left[K, Z_{2}\right] K+Z_{2}\left(1+K^{2}\right)\right\}
\end{aligned}
$$

we obtain

$$
\left(1+Z_{1}+Z_{2} K\right)^{-1}=\left\{\left(1+Z_{1}\right)^{2}+Z_{2}^{2}\right\}^{-1}\left(1+Z_{1}-Z_{2} K\right)+Q(X, Z)
$$

where

$$
\begin{aligned}
& Q(X, Z) \\
& \quad=\left\{\left(1+Z_{1}\right)^{2}+Z_{2}^{2}\right\}^{-1} Z_{2}\left\{\left[K, Z_{1}\right]+\left[K, Z_{2}\right] K+Z_{2}\left(1+K^{2}\right)\right\}\left(1+Z_{1}+Z_{2} K\right)^{-1} .
\end{aligned}
$$


The identity

$$
\begin{aligned}
\left(1+Z_{1}-Z_{2} K\right) & \left\{Y_{1}+\left(1+Y_{2}\right) K\right\} \\
=\left(1+Z_{1}\right) Y_{1} & +Z_{2}\left(1+Y_{2}\right)+\left\{\left(1+Z_{1}\right)\left(1+Y_{2}\right)-Z_{2} Y_{1}\right\} K \\
& -Z_{2}\left\{\left[K, Y_{1}\right]+\left(1+Y_{2}\right)\left(K^{2}+1\right)+\left[K, Y_{2}\right] K\right\}
\end{aligned}
$$

(5.11) and (5.12) lead to the equation

$$
\begin{aligned}
Y_{1 t t}=-\left\{\left(1+Z_{1}\right)^{2}+Z_{2}^{2}\right\}^{-1}\{(1 & \left.+Z_{1}\right) Y_{1}+Z_{2}\left(1+Y_{2}\right) \\
& \left.+\left(\left(1+Z_{1}\right)\left(1+Y_{2}\right)-Z_{2} Y_{1}\right) K\right\} Y_{1 x}+\cdots .
\end{aligned}
$$

Noting that $\left(1+Z_{1}\right) Y_{1}+Z_{2}\left(1+Y_{2}\right)=0$ and $K=-i \operatorname{sgn} D+i(\operatorname{sgn} D-\tanh (h D))$ $+K_{1}$, and replacing $Y_{2 t}, Z_{1 t}$ and $Z_{2 t}$ in the above equation by $f_{2}, f_{3}$ and $f_{4}$, respectively, we can write the equation for $Y_{1}$ in the form

$$
Y_{1 t t}=-a(-i \operatorname{sgn} D) Y_{1 x}+f_{1}\left(W, W_{t}^{\prime}\right)=-a|D| Y_{1}+f_{1}\left(W, W_{t}^{\prime}\right)
$$

where $a=\left\{\left(1+Z_{1}\right)\left(1+Y_{2}\right)-Z_{2} Y_{1}\right\}\left\{\left(1+Z_{1}\right)^{2}+Z_{2}^{2}\right\}^{-1}$.

Remark 5.14. Since $Y=X_{t t}$ and $Z=X_{x}$, we observe that

$$
\begin{aligned}
\{(1+ & \left.\left.Z_{1}\right)^{2}+Z_{2}^{2}\right\}^{1 / 2} a \\
& =\left\{\left(1+X_{1 x}\right)^{2}+X_{2 x}^{2}\right\}^{-1 / 2}\left(-X_{2 x}, 1+X_{1 x}\right) \cdot\left(X_{1 t t}, 1+X_{2 t t}\right) \\
& =N \cdot\left(X_{t t}+(0,1)\right)=-N \cdot \operatorname{grad} p .
\end{aligned}
$$

Therefore $\left\{\left(1+Z_{1}\right)^{2}+Z_{2}^{2}\right\}^{1 / 2} a$ is the gradient of the pressure in the inner normal direction on the free surface.

Remark 5.15. If $X, Y, Z$ satisfy the equations (5.8), (5.13) and $\left(1+Z_{1}\right) Y_{1}$ $+Z_{2}\left(1+Y_{2}\right)=0$, then the equation (5.11) holds.

The required quasilinear system has the form

$$
\left\{\begin{array}{l}
X_{t t}=Y \\
Y_{1 t t}+a(W)|D| Y_{1}=f_{1}\left(W, W_{t}^{\prime}\right) \\
Y_{2 t}=f_{2}\left(W, W_{t}^{\prime}\right), Z_{1 t}=f_{3}\left(W, W_{t}^{\prime}\right), Z_{2 t}=f_{4}\left(W, W_{t}^{\prime}\right)
\end{array}\right.
$$

where

$$
\left\{\begin{array}{l}
a(W)=a(Y, Z)=\left\{\left(1+Z_{1}\right)\left(1+Y_{2}\right)-Z_{2} Y_{1}\right\}\left\{\left(1+Z_{1}\right)^{2}+Z_{2}^{2}\right\}^{-1} \\
f_{2}=K Y_{1 t}+F_{20}\left(X, X_{t}, Y\right) \\
f_{3}=-\left\{\left(1+Y_{2}\right)(-i \operatorname{sgn} D)+Y_{1}\right\}^{-1}\left\{\left(1+Y_{2}\right) F_{010}\left(X, Z, X_{1 t}\right)\right. \\
\left.\quad+\left(1+Z_{1}\right) Y_{1 t}+Z_{2} f_{2}\left(W, W_{t}^{\prime}\right)\right\}
\end{array}\right.
$$




$$
\left\{\begin{aligned}
F_{010} & =i(\operatorname{sgn} D-\tanh (h D)) \frac{\partial}{\partial x} X_{1 t}+K_{1} \frac{\partial}{\partial x} X_{1 t}+F_{01}\left(X, Z, X_{1 t}\right), \\
f_{4}= & -i \operatorname{sgn} D f_{3}\left(W, W_{t}^{\prime}\right)+F_{010}\left(X, Z, X_{1 t}\right), \\
f_{1}= & -a(W)\left(i \operatorname{sgn} D-i \tanh (h D)+K_{1}\right) \frac{\partial}{\partial x} Y_{1} \\
& +Z_{2}\left\{\left(1+Z_{1}\right)^{2}+Z_{2}^{2}\right\}^{-1}\left\{\left[K, Y_{1}\right]+\left[K, Y_{2}\right] K+\left(1+Y_{2}\right)\left(K^{2}+1\right)\right\} \\
& \frac{\partial}{\partial x} Y_{1} \\
& -Z_{2}\left\{\left(1+Z_{1}\right)^{2}+Z_{2}^{2}\right\}^{-1}\left\{\left[K, Z_{1}\right]+\left[K, Z_{2}\right] K+Z_{2}\left(K^{2}+1\right)\right\} \\
& \times\left(1+Z_{1}+Z_{2} K\right)^{-1}\left\{Y_{1}+\left(1+Y_{2}\right) K\right\} \frac{\partial}{\partial x} Y_{1} \\
& -\left(1+Z_{1}+Z_{2} K\right)^{-1}\left\{\left(1+Y_{2}\right) F_{11}\left(X, X_{t}, Z, Z_{t}, Y_{1}\right)\right. \\
& \left.+Z_{2} F_{30}\left(X, X_{t}, Y, Y_{t}\right)+2 Y_{t} \cdot Z_{t}\right\},
\end{aligned}\right.
$$

(in the last term $Y_{2 t}, Z_{1 t}$ and $Z_{2 t}$ are replaced by $f_{2}, f_{3}$ and $f_{4}$, respectively),

$K=K(X)=-i \tanh (h D)+K_{1}(X)$.

Though $f$ depends on $W, W_{t}^{\prime}, b$ and $h$, we omit $b$ and $h$ in the notations.

5.2. Properties of $\boldsymbol{a}(\boldsymbol{W})$ and $\boldsymbol{f}\left(\boldsymbol{W}, W_{\imath}^{\prime}\right)$. In solving the initial value problem for the system (5.16) we need only the properties of $a$ and $f$ which will be shown in this article. The explicit form (5.17) of $a$ and $f$ will play an important role in dealing with the original problem (1.6) and (1.7).

Lemma 5.18. There exists $c_{0}>0$ such that if $s \geqq 2, d_{0}>0$ and

$$
W=(0, Y, Z) \in H^{s},\|W\|_{2} \leqq c_{0},\|W\|_{s} \leqq d_{0},
$$

then for $a=a(W)=a(Y, Z)$

1) $a^{\prime} \equiv a-1 \in H^{s}$

2) $\left|a^{\prime}\right| \leqq e_{3}<1$, i.e., $0<e_{1} \leqq a \leqq e_{2}$

3) $\left\|\left[a,|D|^{1 / 2}\right] u\right\| \leqq C_{1}\|u\|_{-1 / 2}, \quad u \in H^{-1 / 2}$

4) $\|[a,|D|] u\| \leqq C_{2}\|u\|, \quad u \in H^{0}$

5) $\left\|\left[a,(1+|D|)^{t}\right] u\right\| \leqq C_{3}\|u\|_{t-1}, \quad u \in H^{t-1}, 2 \leqq t \leqq s$

6) for $W^{0}=\left(0, Y^{0}, Z^{0}\right)$ satisfying the condition (5.19)

$$
\left\|a(W)-a\left(W^{0}\right)\right\|_{s} \leqq C\left\|W-W^{0}\right\|_{s}
$$

where $e_{j}=e_{j}\left(c_{0}\right)>0, j=1,2,3, C_{j}=C_{j}\left(c_{0}\right)>0, j=1,2, C_{3}=C_{3}\left(c_{0}, d_{0}, s\right)>0$ and $C=C\left(c_{0}, d_{0}, s\right)>0$.

Proof. From Lemma 2.5 it follows that 


$$
|W(x)| \leqq C\|W\|_{1} \leqq C c_{0} .
$$

Since $a(0)=1$, we can choose $c_{0}$ so small that $a(W)$ is everywhere defined, i.e., $\left(1+Z_{1}\right)^{2}+Z_{2}^{2}>0$ and 2) holds for some $e_{j}$. Let $\Omega \subset \mathbb{R}^{4}$ be the closed ball of radius $C c_{0}$ with the center at the origin. Since

$$
a(W)-a\left(W^{0}\right)=\sum_{3}^{6}\left(W_{j}-W_{j}^{0}\right) \int_{0}^{1} \frac{\partial a}{\partial w_{j}}\left(t W+(1-t) W^{0}\right) d t
$$

it follows from Lemma 2.11 and Remark 2.12 that

$$
\left\|a(W)-a\left(W^{0}\right)\right\|_{r} \leqq C\|a\|_{\mathscr{g}^{m+1}(\Omega)}\left(1+\|W\|_{r}+\left\|W^{0}\right\|_{r}\right)^{m+1}\left\|W-W^{0}\right\|_{r}, \quad 2 \leqq r \leqq s \leqq m .
$$

This proves 6). Putting $W^{0}=0$ we see that $a^{\prime}=a-1 \in H^{s}$ in virtue of $a(0)=1$. Noting that $[a, P(D)]=\left[a^{\prime}, P(D)\right]$ and $\left\|a^{\prime}\right\|_{2} \leqq C,\left(C\right.$ depends only on $\left.c_{0}\right)$, we see by Lemma 2.14 that 3 ), 4) and 5) are valid. The proof is complete.

Lemma 5.20. Let $c_{0}$ be the constant chosen in Lemma 5.18, $0<T<\infty$, $s \geqq 2$ and

$$
\begin{aligned}
& W=(0, Y, Z) \in C^{1}\left([0, T], H^{s}\right), \\
& \|W(t)\|_{2} \leqq c_{0},\|W(t)\|_{s} \leqq d_{0},\left\|W_{t}(t)\right\|_{2} \leqq d, \quad 0 \leqq t \leqq T .
\end{aligned}
$$

Then

1) $a-1 \in C^{1}\left([0, T], H^{s}\right)$.

2) $\left|a_{t}\right| \leqq C_{4} d$.

3) $\left\|\left[a_{t},|D|^{1 / 2}\right] u\right\| \leqq C_{5} d\|u\|_{-1 / 2}, \quad u \in H^{-1 / 2}$,

where $a_{t}=(\partial / \partial t) a(W), C_{j}=C_{j}\left(c_{0}\right)>0, j=4,5$.

Proof. It follows from Lemma 5.18, 6) that $\left\|a(W(t))-a\left(W\left(t_{0}\right)\right)\right\|_{s} \leqq C \| W(t)$ $-W\left(t_{0}\right) \|_{s}$, which proves that $a-1 \in C^{0}\left([0, T], H^{s}\right)$. Note that $a_{t}(W(t))$ $=\sum_{3}^{6} \partial a(W(t)) / \partial W_{j} \cdot \partial W_{j}(t) / \partial t$. This gives

$$
\left|a_{t}(W(t))\right| \leqq\left(\sup \left|\frac{\partial a}{\partial W_{j}}\right|\right)\left|W_{t}\right| \leqq C\left\|W_{t}\right\|_{1} \leqq C_{4} d
$$

and

$$
\left\|a_{t}\right\|_{r} \leqq C\|a\|_{\mathscr{B}^{m+2}(\Omega)}\left(1+\|W(t)\|_{r}\right)^{m+1}\left\|W_{t}(t)\right\|_{r}, \quad 2 \leqq r \leqq s \leqq m ，
$$

in virtue of Lemma 2.11 and Remark 2.12. Using Lemma 2.14, 4) we have

$$
\left\|\left[a_{t},|D|^{1 / 2}\right] u\right\| \leqq C\left\|a_{t}\right\|_{2}\|u\|_{-1 / 2} \leqq C_{5} d\|u\|_{-1 / 2} \text { 。 }
$$

Since 


$$
\begin{aligned}
a_{t}(W(t))-a_{t}\left(W\left(t_{0}\right)\right)= & \sum_{3}^{6} \frac{\partial a}{\partial W_{j}}(W(t))\left\{\frac{\partial W_{j}}{\partial t}(t)-\frac{\partial W_{j}}{\partial t}\left(t_{0}\right)\right\} \\
& +\sum_{3}^{6}\left\{\frac{\partial a}{\partial W_{j}}(W(t))-\frac{\partial a}{\partial W_{j}}\left(W\left(t_{0}\right)\right)\right\} \frac{\partial W_{j}}{\partial t}\left(t_{0}\right)
\end{aligned}
$$

we have $\left\|a_{t}(W(t))-a_{t}\left(W\left(t_{0}\right)\right)\right\|_{s} \leqq C\left\|W(t)-W\left(t_{0}\right)\right\|_{s}$, which proves that $a_{t} \in$ $C^{0}\left([0, T], H^{s}\right)$. The proof is complete.

Lemma 5.22. There exists a small positive constant $c_{0}$ such that if $m$ is an integer $\geqq 3,0<T<\infty, d_{0}>0$ and $b \in H^{m+1},\|b\|_{3} \leqq c_{0},\|b\|_{m+1} \leqq d_{0}$,

$$
\begin{aligned}
W, W_{t}^{\prime} \in C^{0}\left([0, T], H^{m}\right), \quad\|W(t)\|_{3} \leqq c_{0}, \\
\|W(t)\|_{m} \leqq d_{0},\left\|W_{t}^{\prime}(t)\right\|_{m} \leqq d_{0}, \quad 0 \leqq t \leqq T,
\end{aligned}
$$

then

1) $f=f\left(W, W_{t}^{\prime}\right)=f\left(W, W_{t}^{\prime}, b\right) \in C^{0}\left([0, T], H^{m}\right)$,

$$
\left\|f\left(W, W_{t}^{\prime}\right)\right\|_{m} \leqq k\left(\|W\|_{m}^{2}+\left\|W_{t}^{\prime}\right\|_{m}^{2}\right)^{1 / 2} \text {, }
$$

2) for $W^{0}, W_{t}^{0^{\prime}}$ satisfying (5.23),

$$
\left\|f\left(W, W_{t}^{\prime}\right)-f\left(W^{0}, W_{t}^{0^{\prime}}\right)\right\|_{m} \leqq C\left(\left\|W-W^{0}\right\|_{m}+\left\|W_{t}^{\prime}-W_{t}^{0^{\prime}}\right\|_{m}\right)
$$

where $k=k\left(c_{0}, d_{0}, m\right)>0$ and $C=C\left(c_{0}, d_{0}, m\right)>0$.

Proof. We see from (5.2) and (5.17) that

$$
\begin{aligned}
f_{2} & =K(X) Y_{1 t}+F_{20}\left(X, X_{t}, Y\right) \\
& =K(X, b ; X, b) Y_{1 t}+2 K_{1,1,0}\left(X, X_{t}, b ; X, b\right) Y_{1}+K_{1,2,0}\left(X, X_{t}, Y, b ; X, b\right) X_{1 t} .
\end{aligned}
$$

Hence Lemma 4.27 shows that 1) and 2) are valid for $f_{2}$. As to

$$
\begin{aligned}
f_{3}=-\left\{\left(1+Y_{1}\right)(-i \operatorname{sgn} D)+Y_{2}\right\}^{-1} \\
\times\left\{\left(1+Y_{2}\right) F_{010}\left(X, Z, X_{1 t}\right)+\left(1+Y_{1}\right) Y_{1 t}+Z_{2} f_{2}\right\}
\end{aligned}
$$

first of all, note that $\left\{\left(1+Y_{1}\right)(-i \operatorname{sgn} D)+Y_{2}\right\}^{-1}=i \operatorname{sgn} D\left(1+Y_{1}+Y_{2} i \operatorname{sgn} D\right)^{-1}$ $\in L(m, m ; 1)$ by Lemma 4.22 . Since

$$
\begin{aligned}
F_{010}=\{i \operatorname{sgn} D-i \tanh (h D)+ & \left.K_{1}(X, b ; X, b)\right\} \frac{\partial}{\partial x} X_{1 t} \\
& +K_{1,0,1}\left(X, Z, b, b_{x} ; X, b\right) X_{1 t},
\end{aligned}
$$

we see by Lemmas 2.15 and 4.27 that $F_{010}$ has the properties 1) and 2). Since $H^{m}$ is an algebra, we see in virtue of Definition 4.21 that 1) and 2) hold for $f_{3}$. Similarly 1) and 2) hold for $f_{4}=-i \operatorname{sgn} D f_{3}+F_{010}$. For the operators occurring in the definition of $f_{1}$ we see that

$$
\left.a(Y, Z), Z_{2}\left\{\left(1+Z_{1}\right)^{2}+Z_{2}^{2}\right\}^{-1} \in L(m, m ; 1) \text { by } 4.22,3\right) ;
$$


$\left\{i \operatorname{sgn} D-i \tanh (h D)+K_{1}(X, b ; X, b)\right\} \frac{\partial}{\partial x} \in L(3, m ; 3)$ by 2.15 and 4.27;

$\left[K, Y_{1}\right]=-i\left[\operatorname{sgn} D, Y_{1}\right]+\left[i \operatorname{sgn} D-i \tanh (h D)+K_{1}(X, b ; X, b), Y_{1}\right]$ $\in L_{0}(2, m ; 3)$ by $2.14,2.15$ and 4.27 ;

$$
\begin{aligned}
1+K(X, b ; X, b)^{2} & =1+\left\{-i \tanh (h D)+K_{1}\right\}^{2} \\
& =\left\{1-\tanh ^{2}(h D)\right\}-i \tanh (h D) K_{1}-i K_{1} \tanh (h D)+K_{1}^{2} \\
& \in L(2, m ; 3) \text { by } 2.15 \text { and } 4.27
\end{aligned}
$$

$\left\{1+Z_{1}+Z_{2} K\left(X, b, b_{x} ; X, b, b_{x}\right)\right\}^{-1} \in L(2,2 ; 2)$ by 4.25 ;

$\left\{1+Z_{1}+Z_{2} K(X, b ; X, b)\right\}^{-1} \in L(m, m ; 3)$ by 4.28 .

These combined with the already proved properties of $f_{2}, f_{3}$ and $f_{4}$ show that 1 ) and 2) hold for $f_{1}$. The proof is finished.

5.3. Transformation of Initial Values. Suppose that $X$ is a solution of the initial value problem:

$$
\begin{aligned}
& X_{2 t}=K(X) X_{1 t},\left(1+X_{1 x}\right) X_{1 t t}+X_{2 x}\left(1+X_{2 t t}\right)=0, \quad t \geqq 0, \\
& X=U, X_{1 t}=V, \quad t=0 .
\end{aligned}
$$

We shall determine the initial values of $W=(X, Y, Z)$ and $W_{t}^{\prime}=\left(X_{t}, Y_{1 t}\right)$ at $t=0$ for the system (5.16) from $U$ and $V$ by means of (5.4) and (5.24). Since $X_{2 t}$ $=K(X) X_{1 t}$ and $Z=X_{x}$, we put $X_{2 t}=K(U) V$ and $Z=U_{x}, t=0$. From (5.3) with $j=1$ and $k=0$ we obtain

$$
X_{2 t t}=K(X) X_{1 t t}+F_{10}\left(X, X_{t}\right) .
$$

This combined with the second equation in (5.24) shows that

$$
\left(1+X_{1 x}+X_{2 x} K(X)\right) X_{1 t t}=-X_{2 x}-X_{2 x} F_{10}\left(X, X_{t}\right) .
$$

Therefore we put

$$
Y_{1}=-\left\{1+Z_{1}+Z_{2} K(X)\right\}^{-1} Z_{2}\left\{1+F_{10}\left(X, X_{t}\right)\right\}, \quad t=0 .
$$

In view of (5.26) we put $Y_{2}=K(X) Y_{1}+F_{10}\left(X, X_{t}\right), t=0$. In view of $Y=X_{t t}$ and $Y_{2 t}=K(X) Y_{1 t}+F_{20}\left(X, X_{t}, Y\right)$, we have

$$
\begin{aligned}
0 & =\frac{\partial}{\partial t}\left\{\left(1+X_{1 x}\right) Y_{1}+X_{2 x}\left(1+Y_{2}\right)\right\} \\
& =\left(1+X_{1 x}\right) Y_{1 t}+X_{2 x}\left(K Y_{1 t}+F_{20}\right)+Y_{1} X_{1 t x}+\left(1+Y_{2}\right) X_{2 t x} .
\end{aligned}
$$

Hence we put 


$$
\begin{aligned}
Y_{1}=-\left\{1+Z_{1}+Z_{2} K\right\}^{-1} & \left\{Z_{2} F_{20}\left(X, X_{t}, Y\right)\right. \\
+ & \left.Y_{1} \frac{\partial}{\partial x} X_{1 t}+\left(1+Y_{2}\right) \frac{\partial}{\partial x} X_{2 t}\right\}, \quad t=0 .
\end{aligned}
$$

Thus the transformation of $U$ and $V$ into $W$ and $W_{t}^{\prime}, t=0$, is as follows:

$$
\left\{\begin{array}{c}
X=U, \quad X_{1 t}=V, \quad X_{2 t}=K(U) V, \quad Z=U_{x}, \\
Y_{1}=-\left\{1+Z_{1}+Z_{2} K\right\}^{-1} Z_{2}\left\{1+F_{10}\left(X, X_{t}\right)\right\}, \\
Y_{2}=K Y_{1}+F_{10}\left(X, X_{t}\right), \\
Y_{1 t}=-\left\{1+Z_{1}+Z_{2} K\right\}^{-1}\left\{Z_{2} F_{20}\left(X, X_{t}, Y\right)\right. \\
\left.\quad+Y_{1} \frac{\partial}{\partial x} X_{1 t}+\left(1+Y_{2}\right) \frac{\partial}{\partial x} X_{2 t}\right\} .
\end{array}\right.
$$

Remark 5.28. For values of $W$ and $W_{t}^{\prime}$ at $t=0$ defined by (5.27) we have

$$
\begin{aligned}
& \left(1+Z_{1}\right) Y_{1}+Z_{2}\left(1+Y_{2}\right)=0, \\
& \left(1+Z_{1}\right) Y_{1 t}+Z_{2}\left\{K Y_{1 t}+F_{20}\left(X, X_{t}, Y\right)\right\}+Y_{1} \frac{\partial}{\partial x} X_{1 t}+\left(1+Y_{2}\right) \frac{\partial}{\partial x} X_{2 t}=0 .
\end{aligned}
$$

In Section 6 it will be shown that the initial value problem for the system (5.16) is uniquely solvable if the initial values are small. Hence in solving the problem (1.6) and (1.7) we need the following lemma.

Lemma 5.29. There exists $c_{0}>0$ such that if $m$ is an integer $\geqq 3, d_{0}>0$ and

$$
\begin{aligned}
& b \in H^{m}, \quad U \in H^{m+(1 / 2)}, \quad V \in H^{m}, \quad\|b\|_{3} \leqq c_{0}, \quad\|U\|_{3} \leqq c_{0}, \\
& \|b\|_{m}, \quad\|U\|_{m+(1 / 2)}, \quad\|V\|_{m} \leqq d_{0}
\end{aligned}
$$

then by (5.27) $U, V$ are transformed into $W, W_{t}^{\prime}, t=0$, such that

$$
X \in H^{m+(1 / 2)}, \quad X_{t} \in H^{m}, \quad Y, Z \in H^{m-(1 / 2)}, \quad Y_{1 t} \in H^{m-1}
$$

and

$$
\begin{aligned}
& \|X\|_{m+(1 / 2)}+\left\|X_{t}\right\|_{m}+\|Y\|_{m-(1 / 2)}+\|Z\|_{m-(1 / 2)}+\left\|Y_{1 t}\right\|_{m-1} \\
& \quad \leqq C\left(\|U\|_{m+(1 / 2)}+\|V\|_{m}\right)
\end{aligned}
$$

where $C=C\left(c_{0}, d_{0}, m\right)>0$.

Proof. Using Remark 2.10, Lemma 4.27 and the definition of $F_{j 0}$ we obtain the lemma by the same consideration as in the proof of Lemma 5.22. 


\section{§6. Unique Existence Theorems}

In this section we shall show the unique solvability of the initial value problems for the quasilinear system (5.16) and the original (1.6). Throughout this section we assume that every function is real-valued.

6.1. Preliminaries. We use the notations:

$$
\begin{aligned}
& W=(X, Y, Z), \quad W^{\prime}=\left(X, Y_{1}\right), \Lambda=\Lambda(D)=1+|D|, \quad D=\frac{1}{i} \frac{\partial}{\partial x} \\
& a=a(W)=a(Y, Z)=\left\{\left(1+Z_{1}\right)\left(1+Y_{2}\right)-Z_{2} Y_{1}\right\}\left\{\left(1+Z_{1}\right)^{2}+Z_{2}^{2}\right\}^{-1}, \quad a_{t}=\frac{\partial a}{\partial t} .
\end{aligned}
$$

In view of the identity

$$
\begin{aligned}
a|D|= & \frac{1}{2} \Lambda^{-m}(a|D|+|D| a) \Lambda^{m}+\lambda+\frac{1}{2} \Lambda^{-m}(a|D|-|D| a) \Lambda^{m} \\
& -\lambda-\Lambda^{-m}\left[a, \Lambda^{m}\right]|D|
\end{aligned}
$$

we introduce the operators

$$
\left\{\begin{array}{l}
G=G(W)=\frac{1}{2}(a|D|+|D| a)+\lambda \\
G_{m}=G_{m}(W)=\Lambda^{-m} G(W) \Lambda^{m} \\
G_{m}^{\prime}=G_{m}^{\prime}(W)=\lambda-\frac{1}{2} \Lambda^{-m}[a,|D|] \Lambda^{m}+\Lambda^{-m}\left[a, \Lambda^{m}\right]|D| \\
G_{t}=G_{t}(W)=\left[\frac{\partial}{\partial t}, G(W)\right]=\frac{1}{2}\left(a_{t}|D|+|D| a_{t}\right) .
\end{array}\right.
$$

Assumption 6.2. Let $\lambda=1+C_{1}, m$ be an integer $\geqq 2, d_{0}, d>0$,

$$
\begin{aligned}
& W=(0, Y, Z) \in C^{1}\left([0, T], H^{2}\right) \cap C^{0}\left([0, T], H^{m}\right), \\
& \|W(t)\|_{2} \leqq c_{0}, \quad\|W(t)\|_{m} \leqq d_{0}, \quad\left\|W_{t}(t)\right\|_{2} \leqq d, \quad 0 \leqq t \leqq T
\end{aligned}
$$

where $c_{0}, C_{1}=C_{1}\left(c_{0}\right)$ are constants occurring in Lemma 5.18 .

Lemma 6.3. Under Assumption 6.2,

1) $a|D|=G_{m}-G_{m}^{\prime}$

2) $(G u, v)=(u, G v), \quad\left(G_{m} u, v\right)_{m}=\left(u, G_{m} v\right)_{m}, \quad u, v \in H^{m+1}$

3) $\left(G_{m} u, u_{t}\right)_{m}=\frac{1}{2} \frac{d}{d t}\left(G^{m} \Lambda u, \Lambda^{m} u\right)-\frac{1}{2}\left(G_{t} \Lambda^{m} u, \Lambda^{m} u\right), \quad u \in C^{1}\left([0, T], H^{m+1}\right)$

4) $e_{1}\left\||D|^{1 / 2} u\right\|^{2}+\|u\|^{2} \leqq(G u, u) \leqq e_{4}\left(e_{1}\left\||D|^{1 / 2} u\right\|^{2}+\|u\|^{2}\right), \quad u \in \mathscr{S}$

5) $\left|\left(G_{t} u, u\right)\right| \leqq d\left(C_{4} e_{1}^{-1}+C_{5}\right)(G u, u), \quad u \in \mathscr{S}$ 
6) for $W^{0}=\left(0, Y^{0}, Z^{0}\right)$ satisfying conditions in 6.2 and $-m+1 \leqq s \leqq m$,

$$
\begin{aligned}
& \left\|G_{m}^{\prime} u\right\|_{m} \leqq\left(1+C_{1}+\frac{1}{2} C_{2}+C_{3}\right)\|u\|_{m}, \\
& \left\|\left\{G_{m}^{\prime}(W)-G_{m}^{\prime}\left(W^{0}\right)\right\} u\right\|_{m} \leqq C\left(\left\|Y-Y^{0}\right\|_{m}+\left\|Z-Z^{0}\right\|_{m}\right)\|u\|_{m}, \\
& \|G(W) u\|_{s-1} \leqq C\|u\|_{s}, \\
& \left\|\left\{G(W)-G\left(W^{0}\right)\right\} u\right\|_{s-1} \leqq C\left(\left\|Y-Y^{0}\right\|_{m}+\left\|Z-Z^{0}\right\|_{m}\right)\|u\|_{s}
\end{aligned}
$$

where $e_{j}, C_{j}, j=1,2,3$ are constants occurring in Lemma 5.18, $C_{4}, C_{5}$ in 5.20, $C=C\left(c_{0}, d_{0}, m\right)>0$ and $e_{4}=\max \left\{e_{2} e_{1}^{-1}, 1+2 C_{1}\right\}$.

Proof. Since $a-1 \in C^{1}\left([0, T], H^{1}\right)$ by Lemma $5.20, H^{1}$ is an algebra and $(u, v)_{m}=\left(\Lambda^{m} u, \Lambda^{m} v\right)$, we have 1$\left.), 2\right)$ and 3$)$ in virtue of $(6.1)$. Note that

$$
\begin{aligned}
(G u, u) & =\frac{1}{2}(a|D| u, u)+\frac{1}{2}(|D| a u, u)+\lambda(u, u) \\
& =(a|D| u, u)+\lambda(u, u) \\
& =\left(a|D|^{1 / 2} u,|D|^{1 / 2} u\right)+\left(\left[a,|D|^{1 / 2}\right]|D|^{1 / 2} u, u\right)+\lambda(u, u) .
\end{aligned}
$$

Therefore from Lemma 5.18 we obtain

$$
\begin{aligned}
& (G u, u) \geqq e_{1}\left\||D|^{1 / 2} u\right\|^{2}+\left(\lambda-C_{1}\right)\|u\|^{2}, \\
& (G u, u) \leqq e_{2}\left\||D|^{1 / 2} u\right\|^{2}+\left(\lambda+C_{1}\right)\|u\|^{2} .
\end{aligned}
$$

These prove 4). Similarly we have by Lemma 5.20

$$
\begin{aligned}
\left|\left(G_{t} u, u\right)\right| & =\left|\left(a_{t}|D|^{1 / 2} u,|D|^{1 / 2} u\right)+\left(\left[a_{t},|D|^{1 / 2}\right]|D|^{1 / 2} u, u\right)\right| \\
& \leqq C_{4} d\left\||D|^{1 / 2} u\right\|^{2}+C_{5} d\left\||D|^{1 / 2} u\right\|_{-1 / 2}\|u\| \\
& \leqq d\left(C_{4} e_{1}^{-1}+C_{5}\right)\left(e_{1}\left\||D|^{1 / 2} u\right\|^{2}+\|u\|^{2}\right)
\end{aligned}
$$

Hence we have 5) in virtue of 4). Since $\|u\|_{m}=\left\|\Lambda^{m} u\right\|$, by Lemma 5.18 we have

$$
\begin{aligned}
\left\|G_{m}^{\prime} u\right\|_{m} & \leqq \lambda\|u\|_{m}+\frac{1}{2}\left\|[a,|D|] \Lambda^{m} u\right\|+\left\|\left[a, \Lambda^{m}\right]|D| u\right\| \\
& \leqq \lambda\|u\|_{m}+\frac{1}{2} C_{2}\left\|\Lambda^{m} u\right\|+C_{3}\||D| u\|_{m-1} \\
& \leqq\left(\lambda+\frac{1}{2} C_{2}+C_{3}\right)\|u\|_{m},
\end{aligned}
$$

which proves the first inequality of 6). Using Lemma 2.14, 3) and 4) we have

$$
\begin{aligned}
& \left\|\left\{G_{m}^{\prime}(W)-G_{m}^{\prime}\left(W^{0}\right)\right\} u\right\|_{m} \leqq \frac{1}{2}\left\|\left[a(W)-a\left(W^{0}\right),|D|\right] \Lambda^{m} u\right\| \\
& \quad+\left\|\left[a(W)-a\left(W^{0}\right), \Lambda^{m}\right]|D| u\right\| \leqq C\left\|a(W)-a\left(W^{0}\right)\right\|_{m}\|u\|_{m} .
\end{aligned}
$$

Since $\left\|a(W)-a\left(W^{0}\right)\right\|_{m} \leqq C\left(\left\|Y-Y^{0}\right\|_{m}+\left\|Z-Z^{0}\right\|_{m}\right)$ in virtue of Lemma $\left.5.18,6\right)$, we have the second inequality of 6 ). We obtain the inequalities for $G=$ 
$2^{-1}(a|D|+|D| a)+\lambda$ if we show that

$$
\begin{aligned}
& \|a u\|_{s} \leqq C\|u\|_{s}, \quad\left\|\left\{a(W)-a\left(W^{0}\right)\right\} u\right\|_{s} \leqq C\left\|a(W)-a\left(W^{0}\right)\right\|_{m}\|u\|_{s}, \\
& -m \leqq s \leqq m \text {. }
\end{aligned}
$$

Note that $a^{\prime}=a-1 \in H^{m}$, (Lemma 5.18) and $H^{s}, s>1 / 2$, is an algebra, (Remark 2.10). For $1 / 2<s \leqq m$ we have

$$
\begin{aligned}
& \|a u\|_{s} \leqq C\left(1+\left\|a^{\prime}\right\|_{s}\right)\|u\|_{s} \leqq C\left(1+\left\|a^{\prime}\right\|_{m}\right)\|u\|_{s}, \\
& \left\|\left\{a(W)-a\left(W^{0}\right)\right\} u\right\|_{s} \leqq C\left\|a(W)-a\left(W^{0}\right)\right\|_{s}\|u\|_{s} .
\end{aligned}
$$

For $0 \leqq s \leqq 1 / 2$, from Lemma 2.14 it follows that

$$
\begin{aligned}
\|a u\|_{s} & \leqq C\left\|\left(1+|D|^{s}\right) a u\right\| \leqq C\|a u\|+C\left\|\left[|D|^{s}, a\right] u\right\|+C\left\|a|D|^{s} u\right\| \\
& \leqq C(\sup |a|)\|u\|+C\left\|a^{\prime}\right\|_{2}\|u\|_{s-1}+C(\sup |a|)\|u\|_{s} \\
& \leqq C\left(1+\left\|a^{\prime}\right\|_{m}\right)\|u\|_{s}
\end{aligned}
$$

and in the same way

$$
\left\|\left\{a(W)-a\left(W^{0}\right)\right\} u\right\|_{s} \leqq C\left\|a(W)-a\left(W^{0}\right)\right\|_{m}\|u\|_{s} .
$$

For $-m \leqq s<0$, by the above results we have

$$
|(a u, v)|=|(u, a v)| \leqq\|u\|_{s}\|a v\|_{-s} \leqq C\|u\|_{s}\left(1+\left\|a^{\prime}\right\|_{m}\right)\|v\|_{-_{s}} .
$$

Hence the duality between $H^{s}$ and $H^{-s}$ shows that

$$
\|a u\|_{s} \leqq C\left(1+\left\|a^{\prime}\right\|_{m}\right)\|u\|_{s} .
$$

It is easily seen that

$$
\left\|\left\{a(W)-a\left(W^{0}\right)\right\} u\right\|_{s} \leqq C\left\|a(W)-a\left(W^{0}\right)\right\|_{m}\|u\|_{s} .
$$

Thus the proof is finished.

6.2. Linear Equations. Consider the initial value problem:

$$
\begin{aligned}
& u_{t t}-\varepsilon^{2} u_{x x}+A(t) u=f, \quad 0 \leqq t \leqq T \\
& u=u_{0}, u_{t}=u_{1}, \quad t=0 .
\end{aligned}
$$

Let $B, C$ be Banach spaces. We denote by $\mathscr{L}(B, C)$ the Banach space consisting of linear continuous operators from $B$ to $C$.

Lemma 6.6. Let $0<\varepsilon, 0<T<\infty,-\infty<s<+\infty$ and $A \in C^{0}([0, T]$, $\left.\mathscr{L}\left(H^{s+1}, H^{s}\right)\right)$. If $u_{0} \in H^{s+1}, u_{1} \in H^{s}$ and $f \in C^{0}\left([0, T], H^{s}\right)$, then there exists the unique solution $u$ of (6.4), (6.5) such that

$$
u \in C^{j}\left([0, T], H^{s+1-j}\right), \quad j=0,1,2 .
$$


Proof. We shall obtain $u$ as the limit of the sequence $u^{j}, j \geqq 0$, such that $u^{0}=0$ and $u^{j}, j \geqq 1$, is a solution of

$$
\begin{aligned}
& u_{t t}^{j}-\varepsilon^{2} u_{x x}^{j}=-A(t) u^{j-1}+f, \quad 0 \leqq t \leqq T \\
& u^{j}=u_{0}, \quad u_{t}^{j}=u_{1}, \quad t=0
\end{aligned}
$$

Since the solution $v$ of $v_{t t}-\varepsilon^{2} v_{x x}=g$ can be written in the form

$$
\hat{v}(t)=(\cos \varepsilon|\xi| t) \hat{v}(0)+\frac{\sin \varepsilon|\xi| t}{\varepsilon|\xi|} \hat{v}_{t}(0)+\int_{0}^{t} \frac{\sin \varepsilon|\xi|(t-s)}{\varepsilon|\xi|} \hat{g}(s) d s,
$$

it is clear that

$$
\|v(t)\|_{s+1}+\left\|v_{t}(t)\right\|_{s} \leqq C\left\{\|v(0)\|_{s+1}+\left\|v_{t}(0)\right\|_{s}+\int_{0}^{t}\|g(t)\|_{s} d t\right\}, \quad 0 \leqq t \leqq T .
$$

These combined with the assumption on $A(t)$ show that $u^{j}$ is defined and converges to $u$, which is the required solution. The uniqueness of $u$ is easily proved. The proof is complete.

Next consider the initial value problem:

$$
\begin{aligned}
& u_{t t}+G_{m}(W) u=f, \quad 0 \leqq t \leqq T, \\
& u=u_{0}, \quad u_{t}=u_{1}, \quad t=0 .
\end{aligned}
$$

Lemma 6.9. Let $m$ be an integer $\geqq 2$ and the assumption 6.2 hold. If $u_{0} \in H^{m+(1 / 2)}, u_{1} \in H^{m}, f \in C^{0}\left([0, T], H^{m}\right)$ then there exists the unique solution $u$ of (6.7), (6.8) such that

$$
u \in C^{j}\left([0, T], H^{m+(1 / 2)-(j / 2)}\right), \quad j=0,1,2 .
$$

Moreover $u$ satisfies the estimate

$$
|u(t)|_{m} \leqq e^{C t} \sqrt{e_{4}}|u(0)|_{m}+\int_{0}^{t} e^{c(t-s)}\|f(s)\|_{m} d s
$$

where $C=2^{-1} d\left(C_{4} e_{1}^{-1}+C_{5}\right)$ and

$$
|u(t)|_{m}^{2}=\left\|u_{t}\right\|_{m}^{2}+e_{1}\left\||D|^{1 / 2} u\right\|_{m}^{2}+\|u\|_{m}^{2} .
$$

Proof. The proof is divided into three steps.

Step 1: Let $u \in C^{2}\left([0, T], H^{m+1}\right)$ satisfy the equation (6.7) and put

$$
E_{m}(u(t))^{2}=\left\|u_{t}\right\|_{m}^{2}+\left(G \Lambda^{m} u, \Lambda^{m} u\right) .
$$

Then

$$
\frac{1}{2} \frac{d}{d t} E_{m}(u(t))^{2}=\left(u_{t t}, u_{t}\right)_{m}+\left(G \Lambda^{m} u, \Lambda^{m} u_{t}\right)+\frac{1}{2}\left(G_{t} \Lambda^{m} u, \Lambda^{m} u\right)
$$




$$
\begin{aligned}
& =\left(f, u_{t}\right)_{m}+\frac{1}{2}\left(G_{t} \Lambda^{m} u, \Lambda^{m} u\right) \\
& \leqq\|f\|_{m}\left\|u_{t}\right\|_{m}+\frac{1}{2} d\left(C_{4} e_{1}^{-1}+C_{5}\right)\left(G \Lambda^{m} u, \Lambda^{m} u\right)
\end{aligned}
$$

in virtue of Lemma 6.3. Therefore for $t$ such that $E_{m}(u(t))>0$,

$$
\frac{d}{d t} E_{m}(u(t)) \leqq\|f\|_{m}+C E_{m}(u(t)) .
$$

Since $E_{m}(u(t))$ is continuous in $t$ we have

$$
E_{m}(u(t)) \leqq e^{C t} E_{m}(u(0))+\int_{0}^{t} e^{c(t-s)}\|f(s)\|_{m} d s .
$$

Using $|u(t)|_{m} \leqq E_{m}(u(t)) \leqq \sqrt{e_{4}}|u(t)|_{m}$ (see Lemma 6.3, 4)), we obtain (6.10). Let $u$ be the solution stated in this lemma. Since $\varphi_{\delta} * u \in C^{2}\left([0, T], H^{m+1}\right)$ and

$$
\left(\varphi_{\delta} * u\right)_{t t}+G_{m}\left(\varphi_{\delta} * u\right)=\varphi_{\delta} * f-\varphi_{\delta} * G_{m} u+G_{m}\left(\varphi_{\delta} * u\right),
$$

$\varphi_{\delta} * u$ satisfies the estimate obtained in the above if we replace $f$ by the right-hand side of (6.11). By Lemma 2.3

$$
\left\|\varphi_{\delta} * u-u\right\|_{m+(1 / 2)}+\left\|\left(\varphi_{\delta} * u\right)_{t}-u_{t}\right\|_{m} \rightarrow 0, \quad \delta \rightarrow+0 .
$$

Hence

$$
\begin{aligned}
& \left|\varphi_{\delta} * u(t)\right|_{m} \rightarrow|u(t)|_{m}, \quad \delta \rightarrow+0, \\
& \left\|\varphi_{\delta} * f\right\|_{m} \leqq\|f\|_{m},\left\|\varphi_{\delta} * f-f\right\|_{m} \rightarrow 0, \quad \delta \rightarrow+0 .
\end{aligned}
$$

By the simple calculation we have

$$
\begin{aligned}
& \left\|\varphi_{\delta} * G_{m} u-G_{m}\left(\varphi_{\delta} * u\right)\right\|_{m}=\left\|\Lambda^{m}\left(\varphi_{\delta} * \Lambda^{-m} G \Lambda^{m} u\right)-G \Lambda^{m}\left(\varphi_{\delta} * u\right)\right\| \\
& \quad=\left\|\left[\varphi_{\delta} *, a\right] D \operatorname{sgn} D \Lambda^{m} u+\frac{1}{2} \varphi_{\delta} *[|D|, a] \Lambda^{m} u-\frac{1}{2}[|D|, a]\left(\varphi_{\delta} * \Lambda^{m} u\right)\right\| .
\end{aligned}
$$

Lemmas $2.3,2.4$ and $5.18,4)$ show that this is bounded when $0 \leqq t \leqq T$ and $0<\delta<1$, and tends to zero when $\delta \rightarrow+0$. Consequently we have the estimate (6.10), which assures the uniqueness of $u$.

Step 2: Let $0<\varepsilon<1$ and consider the initial value problem

$$
\begin{aligned}
& v_{t t}-\varepsilon^{2} v_{x x}+G_{m}(W) v=g, \quad 0 \leqq t \leqq T \\
& v=v_{0}, \quad v_{t}=v_{1}, \quad t=0
\end{aligned}
$$

where $v_{0} \in H^{m+2}, v_{1} \in H^{m+1}$ and $g \in C^{0}\left([0, T], H^{m+1}\right)$. It follows from Lemma $6.3,6)$ that

$$
G_{m} \in C^{0}\left([0, T], \mathscr{L}\left(H^{s}, H^{s-1}\right)\right), \quad 1 \leqq s \leqq 2 m
$$


Since $1 \leqq m+2 \leqq 2 m$ in virtue of $m \geqq 2$, putting $s=m+1$ in Lemma 6.6 we see that there exists the unique solution $v=v^{\varepsilon}$ of (6.12) and (6.13) such that

$$
v \in C^{j}\left([0, T], H^{m+2-j}\right), \quad j=0,1,2 .
$$

Put $E_{m+1, \varepsilon}(v(t))^{2}=\left\|v_{t}\right\|_{m+1}^{2}+\varepsilon^{2}\left\|v_{x}\right\|_{m+1}^{2}+\left(G \Lambda^{m+1} v, \Lambda^{m+1} v\right)$. Since $\Lambda^{m+1}\left(G_{m+1}\right.$ $\left.-G_{m}\right)=2^{-1}\left[a,|D|^{2}\right] \Lambda^{m}=2^{-1}\left[a,(1+|D|)^{2}-2|D|\right] \Lambda^{m}$, in virtue of Lemma 5.18 we have

$$
\begin{aligned}
& \left|\left(\Lambda^{m+1}\left(G_{m+1}-G_{m}\right) v, \Lambda^{m+1} v_{t}\right)\right| \\
& \quad \leqq\left(\frac{1}{2}\left\|\left[(1+|D|)^{2}, a\right] \Lambda^{m} v\right\|+\left\|[|D|, a] \Lambda^{m} v\right\|\right)\left\|\Lambda^{m+1} v_{t}\right\| \\
& \quad \leqq\left(\frac{1}{2} C_{3}+C_{2}\right)\|v\|_{m+1}\left\|v_{t}\right\|_{m+1} .
\end{aligned}
$$

We have

$$
\begin{aligned}
\frac{1}{2} & \frac{d}{d t} E_{m+1, \varepsilon}(v(t))^{2} \\
& =\left(v_{t t}, v_{t}\right)_{m+1}+\varepsilon^{2}\left(v_{x}, v_{x t}\right)_{m+1}+\left(G \Lambda^{m+1} v, \Lambda^{m+1} v_{t}\right)+\frac{1}{2}\left(G_{t} \Lambda^{m+1} v, \Lambda^{m+1} v\right) \\
& =\left(v_{t t}-\varepsilon^{2} v_{x x}+G_{m+1} v, v_{t}\right)_{m+1}+\frac{1}{2}\left(G_{t} \Lambda^{m+1} v, \Lambda^{m+1} v\right) \\
& =\left(g+G_{m+1} v-G_{m} v, v_{t}\right)_{m+1}+\frac{1}{2}\left(G_{t} \Lambda^{m+1} v, \Lambda^{m+1} v\right) \\
& \leqq\|g\|_{m+1} E_{m+1, \varepsilon}(v(t))+C E_{m+1, \varepsilon}(v(t))^{2}
\end{aligned}
$$

Therefore

$$
E_{m+1, \varepsilon}(v(t)) \leqq e^{C t} E_{m+1, \varepsilon}(v(0))+\int_{0}^{t} e^{C(t-s)}\|g(s)\|_{m+1} d s
$$

Since

$$
\begin{aligned}
E_{m+1, \varepsilon}(v(0))^{2} & =\left\|v_{1}\right\|_{m+1}^{2}+\varepsilon^{2}\left\|v_{0 x}\right\|_{m+1}^{2}+\left(G \Lambda^{m+1} v_{0}, \Lambda^{m+1} v_{0}\right) \\
& \leqq\left\|v_{1}\right\|_{m+1}^{2}+\left\|v_{0}\right\|_{m+2}^{2}+e_{4}\left(e_{1}\left\||D|^{1 / 2} \Lambda^{m+1} v_{0}\right\|^{2}+\left\|\Lambda^{m+1} v_{0}\right\|^{2}\right),
\end{aligned}
$$

there exists a constant $C$ independent of $0<\varepsilon<1,0 \leqq t \leqq T$ such that

$$
\left\|\varepsilon v_{x}^{e}(t)\right\|_{m+1} \leqq C, \quad 0<\varepsilon<1,0 \leqq t \leqq T .
$$

Let $0<\varepsilon, \delta<1$ and put $v=v^{\varepsilon}-v^{\delta}$. Then

$$
\left\{\begin{array}{l}
v_{t t}+G_{m} v=\varepsilon^{2} v_{x x}^{\varepsilon}-\delta^{2} v_{x x}^{\delta}, \quad 0 \leqq t \leqq T \\
v=0, \quad v_{t}=0, \quad t=0
\end{array}\right.
$$

Applying the estimate (6.10) to $v$, we have

$$
|v(t)|_{m} \leqq e^{C T} \int_{0}^{t}\left\|\varepsilon^{2} v_{x x}^{\varepsilon}-\delta^{2} v_{x x}^{\delta}\right\|_{m} d s \leqq e^{C T} C T(\varepsilon+\delta) .
$$


Hence there exists $u$ such that, when $\varepsilon \rightarrow+0$,

$$
v^{\varepsilon}(t) \rightarrow u(t) \quad \text { in } H^{m+(1 / 2)}, \quad v_{t}^{\varepsilon}(t) \rightarrow u_{t}(t) \quad \text { in } H^{m}
$$

uniformly in $0 \leqq t \leqq T$. These, combined with (6.14) and (6.16), show that

$$
\varepsilon^{2} v_{x x}^{\varepsilon} \rightarrow 0 \quad \text { in } H^{m}, \quad G_{m} v^{\varepsilon} \rightarrow G_{m} u \quad \text { in } H^{m-(1 / 2)}
$$

uniformly in $0 \leqq t \leqq T$. By the equation $v_{t t}^{\varepsilon}=\varepsilon^{2} v_{x x}^{\varepsilon}+G_{m} v^{\varepsilon}+f$, we see that

$$
v_{t t}^{\varepsilon} \rightarrow u_{t t} \text { in } H^{m-(1 / 2)} \text { uniformly in } 0 \leqq t \leqq T .
$$

Consequently $u \in C^{j}\left([0, T], H^{m+(1 / 2)-(j / 2)}\right), j=0,1,2$, and $u$ is the solution of

$$
\left\{\begin{array}{l}
u_{t t}+G_{m} u=g, \quad 0 \leqq t \leqq T \\
u=v_{0}, \quad u_{t}=v_{1}, \quad t=0
\end{array}\right.
$$

Step 3: Let $0<\delta<1$ and put $v_{0}=\varphi_{\delta} * u_{0}, v_{1}=\varphi_{\delta} * u_{1}, g=\varphi_{\delta} * f$. Then by the step 2, there exists the unique solution $u^{\delta}$ of (6.17). Putting $w=u^{\varepsilon}-u^{\delta}$ and applying the estimate (6.10) to $w$, we have

$$
\begin{aligned}
|w(t)|_{m} \leqq & e^{C T}\left\{\sqrt{e_{4}}\left(\left\|\varphi_{\delta} * u_{1}-\varphi_{\varepsilon} * u_{1}\right\|_{m}^{2}+\left(e_{1}+1\right)\left\|\varphi_{\delta} * u_{0}-\varphi_{\varepsilon} * u_{0}\right\|_{m+(1 / 2)}^{2}\right)^{1 / 2}\right. \\
& \left.+\int_{0}^{T}\left\|\varphi_{\delta} * f-\varphi_{\varepsilon} * f\right\|_{m} d s\right\}, \quad 0 \leqq t \leqq T .
\end{aligned}
$$

Lemma 2.3 and the argument as in the step 2 show that when $\delta \rightarrow+0$, the limit $u$ of $u^{\delta}$ exists and $u$ is the required solution, which completes the proof.

Now consider the initial value problem:

$$
\begin{aligned}
& u_{t t}+a(W)|D| u=f, \quad 0 \leqq t \leqq T \\
& u=u_{0}, \quad u_{t}=u_{1}, \quad t=0
\end{aligned}
$$

Theorem 6.20. Under Assumption 6.2, if $u_{0} \in H^{m+(1 / 2)}, u_{1} \in H^{m}$ and $f$ $\in C^{0}\left([0, T], H^{m}\right)$, then there exists the unique solution $u$ of (6.18) and (6.19) such that $u \in C^{j}\left([0, T], H^{m+(1 / 2)-(j / 2)}\right), j=0,1,2$. Moreover $u$ satisfies the estimate

$$
|u(t)|_{m} \leqq \sqrt{e_{4}} e^{C t}|u(0)|_{m}+\int_{0}^{t} e^{C(t-s)}\|f(s)\|_{m} d s
$$

where $|u(t)|_{m}^{2}=\left\|u_{t}\right\|_{m}^{2}+e_{1}\left\||D|^{1 / 2} u\right\|_{m}^{2}+\|u\|_{m}^{2}$ and $C=1+C_{1}+2^{-1} C_{2}+C_{3}+(d / 2)$ $\times\left(C_{4} e_{1}^{-1}+C_{5}\right)$.

Proof. Using $a|D|=G_{m}-G_{m}^{\prime}$ we write (6.18) in the form

$$
u_{t t}+G_{m} u=f+G_{m}^{\prime} u
$$


The solution $u$ will be obtained as the limit of the sequence $u^{j}, j \geqq 0$, such that $u^{0}=0$ and $u^{j}, j \geqq 1$, is a solution of

$$
\left\{\begin{array}{l}
u_{t t}^{j}+G_{m} u^{j}=f+G_{m}^{\prime} u^{j-1} \\
u^{j}=u_{0}, \quad u_{t}^{j}=u_{1}, \quad t=0 .
\end{array}\right.
$$

Since $G_{m}^{\prime} \in C^{0}\left([0, T], \mathscr{L}\left(H^{m}, H^{m}\right)\right)$ by Lemma 6.3 , it follows from Lemma 6.9 that $u^{j}, j \geqq 1$, is uniquely defined and

$$
u^{j} \in C^{k}\left([0, T], H^{m+(1 / 2)-(k / 2)}\right), \quad k=0,1,2 .
$$

By the estimate (6.10) and Lemma 6.3 we have

$$
\left|u^{j+1}(t)-u^{j}(t)\right|_{m} \leqq C \int_{0}^{t}\left\|u^{j}(s)-u^{j-1}(s)\right\|_{m} d s \leqq C \int_{0}^{t}\left|u^{j}(s)-u^{j-1}(s)\right|_{m} d s,
$$

which assures the existence of the required solution $u$. Applying the estimate (6.10) to the solution $u$ of (6.22) we have in virtue of Lemma 6.3

$$
\begin{aligned}
e^{-C_{t}}|u(t)|_{m} \leqq & \sqrt{e_{4}}|u(0)|_{m}+\int_{0}^{t} e^{-C s}\|f(s)\|_{m} d s \\
& +\left(1+C_{1}+\frac{1}{2} C_{2}+C_{3}\right) \int_{0}^{t} e^{-C s}|u(s)|_{m} d s
\end{aligned}
$$

where $C=(d / 2)\left(C_{4} e_{1}^{-1}+C_{5}\right)$. Therefore we have

$$
\begin{aligned}
e^{-C t}|u(t)|_{m} \leqq & \sqrt{e_{4}}|u(0)|_{m} \exp \left(1+C_{1}+\frac{1}{2} C_{2}+C_{3}\right) t \\
& +\int_{0}^{t}\|f(s)\|_{m} e^{-C s} \exp \left\{\left(1+C_{1}+\frac{1}{2} C_{2}+C_{3}\right)(t-s)\right\} d s,
\end{aligned}
$$

which gives (6.21). The proof is complete.

6.3. Quasilinear System. We use the notations:

$$
\begin{gathered}
W=(X, Y, Z), W^{\prime}=\left(X, Y_{1}\right), \quad\left|Y_{1}(t)\right|_{m}^{2}=\left\|Y_{1}\right\|_{m}^{2}+e_{1}\left\||D|^{1 / 2} Y_{1}\right\|_{m}^{2}+\left\|Y_{1 t}\right\|_{m}^{2}, \\
\begin{array}{c}
|W|_{m}^{2}=|W(t)|_{m}^{2}=\|W(t)\|_{m}^{2}+\left\|W_{t}^{\prime}(t)\right\|_{m}^{2}+e_{1}\left\||D|^{1 / 2} Y_{1}\right\|_{m}^{2} \\
=\|X\|_{m}^{2}+\left\|X_{t}\right\|_{m}^{2}+\left\|Y_{1}\right\|_{m}^{2}+e_{1}\left\||D|^{1 / 2} Y_{1}\right\|_{m}^{2}+\left\|Y_{1 t}\right\|_{m}^{2}+\left\|Y_{2}\right\|_{m}^{2}+\|Z\|_{m}^{2} .
\end{array}
\end{gathered}
$$

We shall consider the initial value problem for the quasilinear system (see (5.16)):

$$
\left\{\begin{array}{l}
X_{t t}=Y \\
Y_{1 t t}+a(W)|D| Y_{1}=f_{1} \\
Y_{2 t}=f_{2}, \quad Z_{1 t}=f_{3}, \quad Z_{2 t}=f_{4}
\end{array}\right.
$$

To simplify the notations we write the initial condition at $t=0$ in the form

$$
W(0)=\tilde{W}=(\tilde{X}, \tilde{Y}, \tilde{Z}), \quad W_{t}^{\prime}(0)=\widetilde{W_{t}^{\prime}}=\left(\widetilde{X_{t}}, \widetilde{Y_{1 t}}\right)
$$


Theorem 6.25. Let $c_{0}$ be so small that Lemmas 5.18 and 5.22 hold, $m$ be an integer $\geqq 4$ and $b \in H^{m+1},\|b\|_{3} \leqq c_{0}$. If $\widetilde{W}, \widetilde{W_{t}^{\prime}}$ satisfy the conditions;

$$
\widetilde{W}, \widetilde{W_{t}^{\prime}},|D|^{1 / 2} \widetilde{Y_{1}} \in H^{m} \text { and }\|\widetilde{W}\|_{3}<c_{0},
$$

then we can choose $T>0$ such that the initial value problem (6.23) and (6.24) has the unique solution $W$ such that

$$
\begin{aligned}
& X \in C^{2}\left([0, T], H^{m}\right), Y_{2}, Z \in C^{1}\left([0, T], H^{m}\right), \\
& Y_{1} \in C^{j}\left([0, T], H^{m+(1 / 2)-(j / 2)}\right), \quad j=0,1,2, \quad\|W(t)\|_{3} \leqq c_{0}, \quad 0 \leqq t \leqq T .
\end{aligned}
$$

Proof. The proof is decomposed into several steps.

Step 1: Take $d, d_{0}, d_{1}, d_{3}$ and $d_{m}$ such that

$$
\left\{\begin{array}{l}
\|b\|_{m+1} \leqq d_{0}, \quad\|\tilde{W}\|_{m} \leqq d_{0}, \quad\left\|\widetilde{W_{t}^{\prime}}\right\|_{m} \leqq d_{0}, \quad\left\|\widetilde{W}_{t}^{\prime}\right\|_{3} \leqq d_{1} \\
d_{j}=\left(2+\sqrt{e_{4}}\right)\left\{\|\tilde{W}\|_{j}^{2}+\left\|\widetilde{W}_{t}^{\prime}\right\|_{j}^{2}+e_{1}\left\||D|^{1 / 2} \widetilde{Y}_{1}\right\|_{j}^{2}\right\}^{1 / 2}, \quad j=3, m, \\
d_{3}<d_{1}, \quad d_{m}<d_{0}, \quad d=\left\{d_{1}^{2}+k_{0}^{2}\left(c_{0}^{2}+d_{1}^{2}\right)\right\}^{1 / 2}
\end{array}\right.
$$

where $k_{0}=k\left(c_{0}, \max \left(c_{0}, d_{1}\right), 3\right)$ is the constant occurring in Lemma 5.22. Now we shall estimate the solution $W$ satisfying the conditions,

$$
\|W(t)\|_{3} \leqq c_{0}, \quad\left\|W_{t}^{\prime}(t)\right\|_{3} \leqq d_{1}, \quad\|W(t)\|_{m} \leqq d_{0}, \quad\left\|W_{t}^{\prime}(t)\right\|_{m} \leqq d_{0}, \quad 0 \leqq t \leqq T .
$$

We see that $\|Y(t)\|_{2}^{2}+\|Z(t)\|_{2}^{2} \leqq\|W(t)\|_{3}^{2} \leqq c_{0}^{2},\|Y(t)\|_{m}^{2}+\|Z(t)\|_{m}^{2} \leqq\|W(t)\|_{m}^{2} \leqq d_{0}^{2}$ and in virtue of Lemma 5.22

$$
\begin{aligned}
\left\|Y_{t}(t)\right\|_{2}^{2}+\left\|Z_{t}(t)\right\|_{2}^{2} & \leqq\left\|Y_{1 t}(t)\right\|_{3}^{2}+\left\|\left(Y_{2 t}, Z_{t}\right)\right\|_{3}^{2} \\
& \leqq d_{1}^{2}+\left\|f\left(W, W_{t}^{\prime}\right)\right\|_{3}^{2} \\
& \leqq d_{1}^{2}+k_{0}^{2}\left(c_{0}^{2}+d_{1}^{2}\right)=d^{2} .
\end{aligned}
$$

Therefore we can use (6.21), which gives

$$
\begin{aligned}
\left|Y_{1}(t)\right|_{m} & \leqq \sqrt{e_{4}}\left|Y_{1}(0)\right|_{m} e^{C t}+\int_{0}^{t} e^{C(t-s)}\left\|f_{1}\left(W, W_{t}^{\prime}\right)\right\|_{m} d s \\
& \leqq \sqrt{e_{4}}|W(0)|_{m} e^{C t}+k\left(c_{0}, d_{0}, m\right) \int_{0}^{t} e^{C(t-s)}|W(s)|_{m} d s
\end{aligned}
$$

in virtue of Lemma 5.22. For $Y_{2}, Z$ we have

$$
\begin{aligned}
\left\|\left(Y_{2}(t), Z(t)\right)\right\|_{m} & =\left\|\left(Y_{2}(0), Z(0)\right)+\int_{0}^{t}\left(f_{2}, f_{3}, f_{4}\right) d s\right\|_{m} \\
& \leqq|W(0)|_{m}+k \int_{0}^{t}|W(s)|_{m} d s
\end{aligned}
$$

Since

$$
\frac{1}{2} \frac{d}{d t}\left\{\left(X_{t}, X_{t}\right)_{m}+(X, X)_{m}\right\}=\left(X_{t t}+X, X_{t}\right)_{m}=\left(X+Y, X_{t}\right)_{m}
$$




$$
\leqq\|X+Y\|_{m}\left\|X_{t}\right\|_{m} \leqq 2|W(t)|_{m}\left\|\left(X, X_{t}\right)\right\|_{m},
$$

we have

$$
\left\|\left(X, X_{t}\right)\right\|_{m} \leqq|W(0)|_{m}+2 \int_{0}^{t}|W(s)|_{m} d s
$$

Hence

$$
\begin{aligned}
|W(t)|_{m} & \leqq\left\|\left(X, X_{t}\right)\right\|_{m}+\left\|\left(Y_{2}, Z\right)\right\|_{m}+\left|Y_{1}\right|_{m} \\
& \leqq\left(2+\sqrt{e_{4}}\right)|W(0)|_{m} e^{C t}+(2+2 k) \int_{0}^{t} e^{C(t-s)}|W(s)|_{m} d s,
\end{aligned}
$$

which gives the estimate

$$
|W(t)|_{m} \leqq\left(2+\sqrt{e_{4}}\right)|W(0)|_{m} \exp \left(k_{m} t\right)=d_{m} \exp \left(k_{m} t\right)
$$

where $k_{m}=2+2 k\left(c_{0}, d_{0}, m\right)+1+C_{1}+2^{-1} C_{2}+C_{3}+2^{-1} d\left(C_{4} e_{1}^{-1}+C_{5}\right), \quad C_{3}$ $=C_{3}\left(c_{0}, d_{0}, m\right)$. Replacing $m$ by 3 we have

$$
|W(t)|_{3} \leqq\left(2+\sqrt{e_{4}}\right)|W(0)|_{3} \exp \left(k_{3} t\right)=d_{3} \exp \left(k_{3} t\right)
$$

where $k_{3}=2+2 k_{0}+1+C_{1}+2^{-1} C_{2}+C_{3}+2^{-1} d\left(C_{4} e_{1}^{-1}+C_{5}\right), C_{3}=C_{3}\left(c_{0}, c_{0}, 3\right)$.

Step 2: Put

$$
T=\min \left\{\frac{1}{k_{3}} \log \frac{d_{1}}{d_{3}}, \frac{1}{k_{m}} \log \frac{d_{0}}{d_{m}}, \frac{c_{0}-\|\tilde{W}\|_{3}}{\left(1+k_{0}\right) d_{1}}\right\} .
$$

Since $0 \leqq d_{3}<d_{1}, 0 \leqq d_{m}<d_{0}, 0<c_{0}-\|\tilde{W}\|_{3}, 0<k, k_{3}, k_{m}$ we see that $0<T<\infty$. By $S$ we denote the totality of $W$ satisfying the following conditions:

$$
\begin{aligned}
& W, W_{t},|D|^{1 / 2} Y_{1} \in C^{0}\left([0, T], H^{m}\right), \\
& \left\{\begin{array}{l}
\|W(t)-\tilde{W}\|_{3} \leqq c_{0}-\|\tilde{W}\|_{3}, \quad\left\|W_{t}^{\prime}(t)\right\|_{3} \leqq d_{1}, \quad\|W(t)\|_{m} \leqq d_{0}, \\
\left\|W_{t}^{\prime}(t)\right\|_{m} \leqq d_{0} \\
\left\|\left(Y_{t}(t), Z_{t}(t)\right)\right\|_{3} \leqq d, \quad|W(t)|_{j} \leqq d_{j} \exp \left(k_{j} t\right), \quad j=3, m \\
\text { for } 0 \leqq \iota \leqq T, W(0)=\tilde{W} .
\end{array}\right.
\end{aligned}
$$

We denote by $M\left(W^{0}\right)$ the solution $W$ of the initial value problem,

$$
\begin{aligned}
& \left\{\begin{array}{l}
X_{t t}+X=X^{0}+Y^{0} \\
Y_{1 t t}+a\left(W^{0}\right)|D| Y_{1}=f_{1}\left(W^{0}, W_{t}^{0^{\prime}}\right) \\
Y_{2 t}=f_{2}\left(W^{0}, W_{t}^{0^{\prime}}\right), \quad Z_{1 t}=f_{3}\left(W^{0}, W_{t}^{0^{\prime}}\right), \quad Z_{2 t}=f_{4}\left(W^{0}, W_{t}^{0^{\prime}}\right)
\end{array}\right. \\
& W(0)=\widetilde{W}, \quad W_{t}^{\prime}(0)=\widetilde{W_{t}^{\prime}} .
\end{aligned}
$$

We shall show that if $W^{0} \in S$ then $W=M\left(W^{0}\right) \in S$. Since

$$
\left\|W^{0}\right\|_{3} \leqq\left\|W^{0}-\tilde{W}\right\|_{3}+\|\tilde{W}\|_{3} \leqq c_{0},
$$


it follows from (6.28) that $Y^{0}, Z^{0}$ satisfy Assumption 6.2 and in virtue of Lemma 5.22 the right-hand sides of (6.29) belong to $C^{0}\left([0, T], H^{m}\right)$. Therefore by the integration and Theorem 6.20 we see that the initial value problem (6.29) and (6.30) has the unique solution $W$ satisfying the condition (6.27). In the same way as in the step 1 we obtain

$$
|W(t)|_{j} \leqq d_{j} \exp \left(k_{j} t\right), \quad j=3, m .
$$

By the definition of $T$ we have for $0 \leqq t \leqq T$

Since $W^{0} \in S$, we have

$$
\|W(t)\|_{j}, \quad\left\|W_{t}^{\prime}(t)\right\|_{j} \leqq|W(t)|_{j} \leqq d_{j} \exp \left(k_{j} t\right)\left\{\begin{array}{l}
\leqq d_{0}, j=m \\
\leqq d_{1}, j=3
\end{array}\right.
$$

$$
\begin{gathered}
\left\|Y_{1 t}\right\|_{3}^{2}+\left\|\left(Y_{2 t}, Z_{t}\right)\right\|_{3}^{2} \leqq d_{1}^{2}+\left\|f\left(W^{0}, W_{t}^{0^{\prime}}\right)\right\|_{3}^{2} \\
\leqq \\
\qquad d_{1}^{2}+k_{0}^{2}\left|W^{0}(t)\right|_{3}^{2} \leqq d_{1}^{2}+k_{0}^{2}\left(c_{0}^{2}+d_{1}^{2}\right)=d^{2}, \\
\|W(t)-\widetilde{W}\|_{3} \leqq \int_{0}^{t}\left\|\frac{d}{d t} W(t)\right\|_{3} d t \leqq \int_{0}^{t}\left\{\left\|W_{t}^{\prime}(t)\right\|_{3}+\left\|\left(Y_{2 t}, Z_{t}\right)\right\|_{3}\right\} d t \\
\leqq \int_{0}^{t}\left\{\left\|W_{t}^{\prime}(t)\right\|_{3}+k_{0}\left|W^{0}(t)\right|_{3}\right\} d t \leqq T\left(d_{1}+k_{0} d_{1}\right) \leqq c_{0}-\|\widetilde{W}\|_{3} .
\end{gathered}
$$

Consequently $W \in S$, which means that $M$ is the mapping from $S$ to itself.

Step 3: Put $W^{0}(t)=\tilde{W}, \quad 0 \leqq t \leqq T . \quad$ Then $X_{t}^{0}=Y_{t}^{0}=0,\left\|W^{0}(t)\right\|_{m}=\|\tilde{W}\|_{m}$ $\leqq d_{0}$,

$$
\begin{aligned}
& \left|W^{0}(t)\right|_{j}=\left(\|\widetilde{W}\|_{j}^{2}+e_{1}\left\||D|^{1 / 2} \widetilde{Y_{1}}\right\|_{j}^{2}\right)^{1 / 2} \\
& \leqq\left(2+\sqrt{e_{4}}\right)\left(\|\widetilde{W}\|_{j}^{2}+\left\|\widetilde{W_{t}^{\prime}}\right\|_{j}^{2}+e_{1}\left\||D|^{1 / 2} \widetilde{Y_{1}}\right\|_{j}^{2}\right)^{1 / 2}=d_{j} \leqq d_{j} \exp \left(k_{j} t\right), \quad j=3, m .
\end{aligned}
$$

We see that $W^{0}$ satisfies (6.27) and (6.28), i.e., $W^{0} \in S$. The result of the step 2 shows that $W^{j+1}=M\left(W^{j}\right), j \geqq 0$, are defined and $W^{j} \in S$. Note that $W$ $=W^{j+1}-W^{j}, j \geqq 1$, is the solution of

$$
\left\{\begin{array}{l}
X_{t t}+X=X^{j}-X^{j-1}+Y^{j}-Y^{j-1} \\
Y_{1 t t}+a\left(W^{j}\right)|D| Y_{1}=f_{1}\left(W^{j}, W_{t}^{j^{\prime}}\right)-f_{1}\left(W^{j-1}, W_{t}^{j-1^{\prime}}\right) \\
\quad-\left(a\left(W^{j}\right)-a\left(W^{j-1}\right)\right)|D| Y_{1}^{j} \\
Y_{2 t}=f_{2}\left(W^{j}, W_{t}^{j^{\prime}}\right)-f_{2}\left(W^{j-1}, W_{t}^{j-1^{\prime}}\right) \\
Z_{k t}=f_{2+k}\left(W^{j}, W_{t}^{j^{\prime}}\right)-f_{2+k}\left(W^{j-1}, W_{t}^{j-1^{\prime}}\right), \quad k=1,2 \\
W(0)=0, \quad W_{t}^{\prime}(0)=0
\end{array}\right.
$$

Since $m-1 \geqq 3$ and $W^{j} \in S$, we have in virtue of Lemma 5.18 with $s=m-1$,

$$
\begin{aligned}
& \left\|\left\{a\left(W^{j}\right)-a\left(W^{j-1}\right)\right\}|D| Y_{1}^{j}\right\|_{m-1} \\
& \quad \leqq C\left\|a\left(W^{j}\right)-a\left(W^{j-1}\right)\right\|_{m-1}\left\|Y_{1}^{j}\right\|_{m} \leqq C\left\|W^{j}-W^{j-1}\right\|_{m-1}\left\|W^{j}\right\|_{m} .
\end{aligned}
$$


Therefore, in virtue of Lemma 5.22, we see that $H^{m-1}$-norms of the right-hand sides of (6.31) are smaller than $C\left|W^{j}(t)-W^{j-1}(t)\right|_{m-1}$ where $C$ is independent of $j$. In the same way as in the step 1 , we have

$$
\begin{aligned}
& \left\|\left(Y_{2}(t), Z(t)\right)\right\|_{m-1}+\left\|\left(X(t), X_{t}(t)\right)\right\|_{m-1}+\left|Y_{1}(t)\right|_{m-1} \\
& \quad \leqq C \int_{0}^{t}\left|W^{j}-W^{j-1}\right|_{m-1} d s .
\end{aligned}
$$

Therefore we obtain

$$
\left|W^{j+1}(t)-W^{j}(t)\right|_{m-1} \leqq C \int_{0}^{t}\left|W^{j}(s)-W^{j-1}(s)\right|_{m-1} d s .
$$

This means that there exists $W$ such that

$$
W, W_{t}^{\prime},|D|^{1 / 2} Y_{1} \in C^{0}\left([0, T], H^{m-1}\right), \sup _{0 \leqq t \leqq T}\left|W^{j}(t)-W(t)\right|_{m-1} \rightarrow 0, \quad j \rightarrow \infty .
$$

Noting that $W^{j+1}=M\left(W^{j}\right) \in S$ and letting $j \rightarrow \infty$ we see that $\left(X_{t t}^{j}, Y_{2 t}^{j}, Z_{t}^{j}\right)$ converges in $H^{m-1}$ and $Y_{1 t t}^{j}$ converges in $H^{m-1-(1 / 2)}$. Hence

$$
\begin{aligned}
& \left\{\begin{array}{l}
X_{t t}=Y, \quad Y_{1 t t}+a(W)|D| Y_{1}=f_{1}\left(W, W_{t}^{\prime}\right), \\
Y_{2 t}=f_{2}\left(W, W_{t}^{\prime}\right), \quad Z_{j t}=f_{2+j}\left(W, W_{t}^{\prime}\right), \quad j=1,2,
\end{array}\right. \\
& \left\{\begin{array}{l}
X \in C^{2}\left([0, T], H^{m-1}\right), \quad Y_{2}, Z \in C^{1}\left([0, T], H^{m-1}\right), \\
Y_{1} \in C^{j}\left([0, T], H^{m-1+(1 / 2)-(j / 2)}\right), \quad j=0,1,2, \\
\|W(t)\|_{3} \leqq c_{0}, \quad\left\|\left(Y_{t}(t), Z_{t}(t)\right)\right\|_{3} \leqq d .
\end{array}\right.
\end{aligned}
$$

Step 4: We shall show that $W$ is the solution required in this theorem, i.e., in (6.32) we can replace $m-1$ by $m$. Noting that $W^{j+1}=M\left(W^{j}\right) \in S$ and using Lemma 5.22 we have

$$
\left\{\begin{array}{l}
\left\|W^{j}(t)\right\|_{m} \leqq d_{0}, \quad\left\|W_{t}^{j^{\prime}}(t)\right\|_{m} \leqq d_{0}, \quad\left|W^{i}(t)\right|_{m} \leqq d_{m} \exp \left(k_{m} t\right), \\
\left\|X_{t t}^{j}(t)\right\|_{m}+\left\|Y_{1 t t}^{j}(t)\right\|_{m-(1 / 2)}+\left\|Y_{2 t}^{j}(t)\right\|_{m}+\left\|Z_{t}^{j}(t)\right\|_{m} \leqq C
\end{array}\right.
$$

where $C>0$ is independent of $t$ and $j$. Since any bounded sequence in a Hilbert space is weakly precompact, each sequence occurring in (6.33) has a weaklimit. By the result of the step 3, they have the strong-limits if $m$ is replaced by $m-1$. Hence for any fixed $t$,

$$
W(t), \quad W_{t}^{\prime}(t), \quad Y_{2 t}(t), \quad|D|^{1 / 2} Y_{1}(t), \quad X_{t t}(t), \quad Z_{t}(t) \in H^{m}, \quad Y_{1 t t}(t) \in H^{m-(1 / 2)} .
$$

Taking the inferior limits of sequences in (6.33) we see that

$$
\left\{\begin{array}{l}
\|W(t)\|_{m} \leqq d_{0}, \quad\left\|W_{t}^{\prime}(t)\right\|_{m} \leqq d_{0}, \quad|W(t)|_{m} \leqq d_{m} \exp \left(k_{m} t\right), \\
\left\|X_{t t}(t)\right\|_{m}+\left\|Y_{1 t t}(t)\right\|_{m-(1 / 2)}+\left\|Y_{2 t}(t)\right\|_{m}+\left\|Z_{t}(t)\right\|_{m} \leqq C
\end{array}\right.
$$


For $0 \leqq t_{0}<t \leqq T$, we have

$$
\left\|W^{j}(t)-W^{j}\left(t_{0}\right)\right\|_{m} \leqq \int_{t_{0}}^{t}\left\|W_{t}^{j}(t)\right\|_{m} d t \leqq\left(t-t_{0}\right)\left(d_{0}+2 C\right) .
$$

Taking the inferior limit we have $\left\|W(t)-W\left(t_{0}\right)\right\|_{m} \leqq\left(t-t_{0}\right)\left(d_{0}+2 C\right)$. Hence $W \in C^{0}\left([0, T], H^{m}\right)$. Similarly we see that $X_{t} \in C^{0}\left([0, T], H^{m}\right)$. By $X_{t t}=Y$, we have $X \in C^{2}\left([0, T], H^{m}\right)$. Since $m-1 \geqq 2,(Y, Z)$ satisfies Assumption 6.2 in virtue of (6.32) and (6.34). Hence by Theorem 6.20 there exists $u^{\delta}, \delta>0$, such that

$$
\begin{aligned}
& u_{t t}^{\delta}+a(W)|D| u^{\delta}=f_{1}\left(W, \varphi_{\delta} * W_{t}^{\prime}\right), \quad 0 \leqq t \leqq T, \\
& u^{\delta}(0)=\widetilde{Y_{1}}, \quad u_{t}^{\delta}(0)=\widetilde{Y_{1 t}}, \\
& u^{\delta} \in C^{j}\left([0, T], \quad H^{m+(1 / 2)-(j / 2)}\right), \quad j=0,1,2 .
\end{aligned}
$$

Using (6.21) we have

$$
\begin{aligned}
\left|u^{\varepsilon}(t)-u^{\delta}(t)\right|_{m} & \leqq C \int_{0}^{t}\left\|f_{1}\left(W, \varphi_{\varepsilon} * W_{t}^{\prime}\right)-f_{1}\left(W, \varphi_{\delta} * W_{t}^{\prime}\right)\right\|_{m} d t \\
& \leqq C \int_{0}^{t}\left\|\varphi_{\varepsilon} * W_{t}^{\prime}-\varphi_{\delta} * W_{t}^{\prime}\right\|_{m} d t \rightarrow 0, \quad \varepsilon, \delta \rightarrow+0 .
\end{aligned}
$$

On the other hand,

$$
\begin{aligned}
\left|u^{\delta}(t)-Y_{1}(t)\right|_{m-1} & \leqq C \int_{0}^{t}\left\|f_{1}\left(W, \varphi_{\delta} * W_{t}^{\prime}\right)-f_{1}\left(W, W_{t}^{\prime}\right)\right\|_{m-1} d t \\
& \leqq C \int_{0}^{t}\left\|\varphi_{\delta} * W_{t}^{\prime}-W_{t}^{\prime}\right\|_{m-1} d t \rightarrow 0, \quad \delta \rightarrow+0
\end{aligned}
$$

Therefore $Y_{1} \in C^{j}\left([0, T], H^{m+(1 / 2)-(j / 2)}\right), j=0,1$. Thus we proved that $W$, $W_{t}^{\prime} \in C^{0}\left([0, T], H^{m}\right)$. Consequently, in virtue of Lemma 5.22, $f\left(W, W_{t}^{\prime}\right)$ $\in C^{0}\left([0, T], H^{m}\right)$, by means of which we see that $Y_{1 t t} \in C^{0}\left([0, T], H^{m-(1 / 2)}\right)$ and $Y_{2 t}, Z_{t} \in C^{0}\left([0, T], H^{m}\right)$. The proof is complete.

Remark 6.35. In the step 2 we defined $T$ by

$$
T=\min \left\{\frac{1}{k_{3}} \log \frac{d_{1}}{d_{3}}, \frac{1}{k_{m}} \log \frac{d_{0}}{d_{m}}, \frac{c_{0}-\|\tilde{W}\|_{3}}{\left(1+k_{0}\right) d_{1}}\right\} .
$$

(For $d, d_{0}, d_{1}, d_{3}$ and $d_{m}$ see (6.26) and for $k_{3}, k_{m}$ see the end of the step 1.) Putting $d_{1}=\sqrt{d_{3}}$ we see that $T \rightarrow \infty$ if $d_{3}, d_{m} \rightarrow 0$, i.e. the initial values, $\widetilde{W}, \widetilde{W_{t}^{\prime}}$ tend to zero.

6.4. Nonlinear Equations. Consider the initial value problem:

$$
\begin{aligned}
& \left(1+X_{1 x}\right) X_{1 t t}+X_{2 x}\left(1+X_{2 t t}\right)=0, X_{2 t}=K X_{1 x}, \quad 0 \leqq t \leqq T, \\
& X=U, \quad X_{1 t}=V, \quad t=0 .
\end{aligned}
$$


Theorem 6.38. Take $c_{0}$ be so small that Lemmas 5.18, 5.22 and 5.29 hold. Let $m$ be an integer $\geqq 5$ and $b \in H^{m},\|b\|_{3} \leqq c_{0}$. There exists $\delta>0$ such that if

$$
U \in H^{m+(1 / 2)}, \quad V \in H^{m}, \quad\|U\|_{4+(1 / 2)} \leqq \delta, \quad\|V\|_{4} \leqq \delta
$$

then there exists $T>0$ such that the initial value problem (6.36) and (6.37) has the unique solution

$$
X \in C^{1}\left([0, T], H^{m}\right) \cap C^{2}\left([0, T], H^{m-(1 / 2)}\right) .
$$

Proof. Existence. Define $W, W_{t}^{\prime}, t=0$, by (5.27). Then it follows from Lemma 5.29 that we can take $\delta>0$ so small that $W, W_{t}^{\prime}, t=0$, satisfy the conditions of Theorem 6.25 if $m$ is replaced by $m-1$. Therefore by Theorem 6.25 we have the solution $W$ of the system (6.23) such that

$$
\begin{aligned}
& X \in C^{2}\left([0, T], H^{m-1}\right), \quad Y_{2}, Z \in C^{1}\left([0, T], H^{m-1}\right), \\
& Y_{1} \in C^{j}\left([0, T], H^{m-1+(1 / 2)-(j / 2)}\right), \quad j=0,1,2,
\end{aligned}
$$

for some $T>0$. It is clear that $X$ satisfies (6.37). We have $\left(1+Z_{1}\right) Y_{1}$ $+Z_{2}\left(1+Y_{2}\right)=0, t=0$, by Remark 5.28 and $\partial / \partial t\left\{\left(1+Z_{1}\right) Y_{1}+Z_{2}\left(1+Y_{2}\right)\right\}=0$, $0 \leqq t \leqq T$, by Remark 5.9. Hence $\left(1+Z_{1}\right) Y_{1}+Z_{2}\left(1+Y_{2}\right)=0,0 \leqq t \leqq T$. Since $Y=X_{t t}$, it remains to show that $Z=X_{x}, X_{2 t}=K X_{1 t}, 0 \leqq t \leqq T$, and $X$ has the required differentiability. Since $Y=X_{t t}$ and

$$
Y_{2 t}=f_{2}=K Y_{1 t}+F_{20}\left(X, X_{t}, Y\right),
$$

we have $\left(X_{2 t}-K X_{1 t}\right)_{t t}=0,0 \leqq t \leqq T$. On the other hand, it follows from (5.27) that $X_{2 t}-K X_{1 t}=0,\left(X_{2 t}-K X_{1 t}\right)_{t}=Y_{2}-K Y_{1}-F_{10}\left(X, X_{t}\right)=0, t=0$. Thus $X_{2 t}$ $=K X_{1 t}, 0 \leqq t \leqq T$. Differentiating this, we have

$$
\left\{\begin{array}{l}
Y_{2}=K Y_{1}+F_{10}\left(X, X_{t}\right) \\
Y_{2 t t}=K Y_{1 t t}+F_{30}\left(X, X_{t}, Y, Y_{t}\right) \\
Y_{2 x}=K Y_{1 x}+F_{11}\left(X, X_{t}, X_{x}, X_{x t}, X_{1 t t}\right) \\
X_{2 t x}=K X_{1 t x}+F_{01}\left(X, X_{x}, X_{1 t}\right)
\end{array}\right.
$$

By Remark 5.9,

$$
\begin{aligned}
Z_{2 t} & =-i \operatorname{sgn} D Z_{1 t}+F_{010}\left(X, Z, X_{1 t}\right) \\
& =-i \operatorname{sgn} D Z_{1 t}+\left(i \operatorname{sgn} D-i \tanh (h D)+K_{1}\right) \frac{\partial}{\partial x} X_{1 t}+F_{01}\left(X, Z, X_{1 t}\right) \\
& =-i \operatorname{sgn} D\left(Z_{1}-X_{1 x}\right)_{t}+K \frac{\partial}{\partial x} X_{1 t}+F_{01}\left(X, Z, X_{1 t}\right) .
\end{aligned}
$$

From this we obtain 


$$
Z_{2 t t}=-i \operatorname{sgn} D\left(Z_{1}-X_{1 x}\right)_{t t}+K Y_{1 x}+F_{11}\left(X, X_{t}, Z, Z_{t}, Y_{1}\right)
$$

Using (6.40) we have

$$
\left(Z_{2}-X_{2 x}\right)_{t}=-i \operatorname{sgn} D\left(Z_{1}-X_{1 x}\right)_{t}+F_{01}\left(X, Z, X_{1 t}\right)-F_{01}\left(X, X_{x}, X_{1 t}\right) .
$$

By Remark 5.15,

$$
\begin{aligned}
0 & =\left(1+Z_{1}\right) Y_{1 t t}+Z_{2} K Y_{1 t t}+Z_{2} F_{30}+Y_{1} Y_{1 x}+\left(1+Y_{2}\right) K Y_{1 x}+\left(1+Y_{2}\right) F_{11}+2 Y_{t} Z_{t} \\
& =\left(1+Z_{1}\right) Y_{1 t t}+Z_{2} Y_{2 t t}+Y_{1} Y_{1 x}+\left(1+Y_{2}\right)\left(K Y_{1 x}+F_{11}\right)+2 Y_{t} Z_{t}
\end{aligned}
$$

Since

$$
\begin{aligned}
0 & =\left\{\left(1+Z_{1}\right) Y_{1}+Z_{2}\left(1+Y_{2}\right)\right\}_{t t} \\
& =\left(1+Z_{1}\right) Y_{1 t t}+Z_{2} Y_{2 t t}+Y_{1} Z_{1 t t}+\left(1+Y_{2}\right) Z_{2 t t}+2 Y_{t} Z_{t},
\end{aligned}
$$

we have

$$
\begin{aligned}
0 & =Y_{1}\left(Z_{1 t t}-Y_{1 x}\right)+\left(1+Y_{2}\right)\left(Z_{2 t t}-K Y_{1 x}-F_{11}\right) \\
& =Y_{1}\left(Z_{1}-X_{1 x}\right)_{t t}+\left(1+Y_{2}\right)(-i \operatorname{sgn} D)\left(Z_{1}-X_{1 x}\right)_{t t} \\
& =\left\{Y_{1}+\left(1+Y_{2}\right)(-i \operatorname{sgn} D)\right\}\left(Z_{1}-X_{1 x}\right)_{t t}
\end{aligned}
$$

where we used (6.41). Thus $\left(Z_{1}-X_{1 x}\right)_{t t}=0,0 \leqq t \leqq T$. In virtue of (5.27), $Z_{1}-X_{1 x}=0, t=0$. By Remark 5.28 and (6.39),

$$
\left(1+Z_{1}\right) Y_{1 t}+Z_{2} Y_{2 t}+Y_{1} X_{1 t x}+\left(1+Y_{2}\right) X_{2 t x}=0, \quad t=0 .
$$

On the other hand,

$$
\left\{\left(1+Z_{1}\right) Y_{1}+Z_{2}\left(1+Y_{2}\right)\right\}_{t}=\left(1+Z_{1}\right) Y_{1 t}+Z_{2} Y_{2 t}+Y_{1} Z_{1 t}+\left(1+Y_{2}\right) Z_{2 t}=0 \text {. }
$$

Therefore, $Y_{1}\left(Z_{1}-X_{1 x}\right)_{t}+\left(1+Y_{2}\right)\left(Z_{2}-X_{2 x}\right)_{t}=0, t=0$. Putting $t=0$ in (6.42), we have $\left(Z_{2}-X_{2 x}\right)_{t}=-i \operatorname{sgn} D\left(Z_{1}-X_{1 x}\right)_{t}, t=0$. Thus $\left(Z_{1}-X_{1 x}\right)_{t}=0, t=0$. Consequently, $Z_{1}-X_{1 x}=0,0 \leqq t \leqq T$. Since $Z_{2}-X_{2 x}=0, t=0$, (6.42) gives

$$
Z_{2}-X_{2 x}=\int_{0}^{t}\left\{F_{01}\left(X, Z, X_{1 t}\right)-F_{01}\left(X, X_{x}, X_{1 t}\right)\right\} d t
$$

We have

$$
\left\|Z_{2}-X_{2 x}\right\|_{m-2} \leqq C \int_{0}^{t}\left\|Z_{2}-X_{2 x}\right\|_{m-2} d t
$$

which shows that $Z_{2}-X_{2 x}=0,0 \leqq \imath \leqq T$. Thus we have proved that $X$ satisfies (6.36), (6.37). Since $X, X_{x}=Z \in C^{1}\left([0, T], H^{m-1}\right)$, we see that $X \in C^{1}([0, T]$, $\left.H^{m}\right)$. Since $X_{1 t t}=Y_{1} \in C^{0}\left([0, T], H^{m-(1 / 2)}\right)$ and

$$
\begin{aligned}
X_{2 t t} & =Y_{2}=K(X) Y_{1}+F_{10}\left(X, X_{t}\right) \\
& =K(X, b ; X, b) Y_{1}+K_{1,1,0}\left(X, X_{t}, b ; X, b\right) X_{1 t}
\end{aligned}
$$


we see by Lemma 4.27 that $X_{2 t t} \in C^{0}\left([0, T], H^{m-(1 / 2)}\right)$.

Uniqueness. Put $W=\left(X, X_{t t}, X_{x}\right)$. By the estimate as in the end of the step 2 of the proof of Theorem 6.25 we see that

$$
\begin{aligned}
\|W(t)-W(0)\|_{3} & \leqq \int_{0}^{t}\left\|\frac{d}{d t} W(t)\right\|_{3} d t \\
& \leqq t\left(d_{1}+k_{0} d_{1}\right)<c_{0}-\|W(0)\|_{3}, \quad 0 \leqq t<T,
\end{aligned}
$$

in virtue of the definition of $T$. Let $X^{0}$ be a solution of (6.36), (6.37) and put $W^{0}=\left(X^{0}, X_{t t}^{0}, X_{x}^{0}\right) . \quad$ It is easily seen that if $\left\|W^{0}(t)\right\|_{3} \leqq c_{0}$ then $W^{0}$ is a solution of (6.23) having the properties stated in Theorem 6.25 where $m$ is replaced by $m-1$. Since $W^{0}(0)=W(0),\|W(0)\|_{3}<c_{0}$ we see that $\|W(t)\|_{3} \leqq c_{0}, 0 \leqq t \leqq t_{0}$ for sufficiently small $t_{0}>0$. By Theorem 6.25 we have $W^{0}(t)=W(t), 0 \leqq t \leqq t_{0}$. Since $\left\|W\left(t_{0}\right)\right\|_{3}<c_{0}$, we see that $\left\|W^{0}(t)\right\|_{3} \leqq c_{0}, t_{0} \leqq t \leqq t_{0}+t_{1}$ for small $t_{1}>0$. Hence $W^{0}(t)=W(t), 0 \leqq t \leqq t_{1}$. Repeating this procedure we see that $W^{0}(t)$ $=W(t), 0 \leqq t<T$, i.e., $X^{0}(t)=X(t), 0 \leqq t \leqq T$. The proof is complete.

Remark 6.43. By Remark 6.35 we see that $T \rightarrow \infty$ if $U \rightarrow 0$ in $H^{m+(1 / 2),}$ $V \rightarrow 0$ in $H^{m}$.

\section{Bibliography}

[1] Налимов, В.И., Задача Коши-Пуассона. Динамика сплошной среды, 18 (1974), 104-210.

[2] Ovsjannikov, L. V., To the shallow water theory foundation, Arch. Mech., 26 (1974), 407-422.

[3] Kano, T., et Nishida, T., Sur les ondes de surface de l'eau avec une justification mathématique des équations des ondes en eau peu profonde, J. Math. Kyoto Univ., 19 (1979), 335-370.

[4] Овсянников, Л.В., Нелинейная задача Коши в шкале банаховых пространств, ДАН СССР, 200 (1971), 789-792.

[5] Налимов, В.И., Априорные оценки решений зллиптических уравнений в классе аналитических функций и их приложения к задаче Коши-Пуассона, ДАН CCCP, 189 (1969), 45-48.

[6] Mizohata, S., The theory of partial differential equations, Cambridge Univ. Press, 1973.

[7] Бесов, О.В., Ильин, В.П. и Никольский, С.М., Интегральные представления функций и теоремы вложения, М. Наука, 1975.

[8] Hörmander, L., Linear partial differential operators, Springer, 1969.

[9] Courant, R. and Hilbert, D., Methods of mathematical physics, Vol. 2, Interscience, New York, 1962.

[10] Petrowsky, I. G., Über das Cauchysche Problem für Systeme von partiellen Differentialgleichungen, Mat. Sb., 2 (44) (1937), 815-870. 